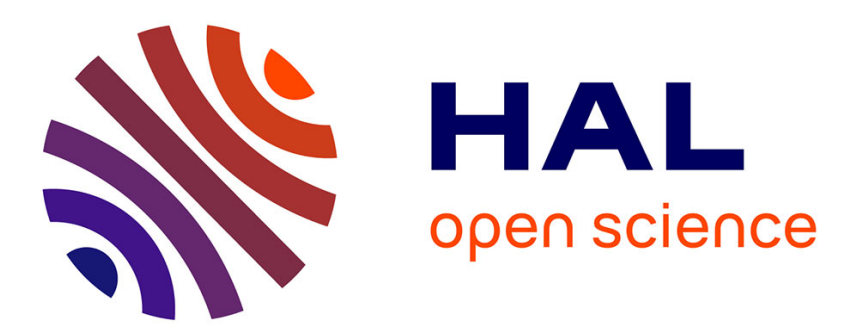

\title{
Optical properties reconstruction using the adjoint method based on the radiative transfer equation
}

\author{
Addoum Ahmad, Olivier Farges, Fatmir Asllanaj
}

\section{To cite this version:}

Addoum Ahmad, Olivier Farges, Fatmir Asllanaj. Optical properties reconstruction using the adjoint method based on the radiative transfer equation. Journal of Quantitative Spectroscopy and Radiative Transfer, 2018, 204, pp.179-189. 10.1016/j.jqsrt.2017.09.015 . hal-01610131

\section{HAL Id: hal-01610131 \\ https://hal.science/hal-01610131}

Submitted on 26 Jan 2018

HAL is a multi-disciplinary open access archive for the deposit and dissemination of scientific research documents, whether they are published or not. The documents may come from teaching and research institutions in France or abroad, or from public or private research centers.
L'archive ouverte pluridisciplinaire HAL, est destinée au dépôt et à la diffusion de documents scientifiques de niveau recherche, publiés ou non, émanant des établissements d'enseignement et de recherche français ou étrangers, des laboratoires publics ou privés. 


\title{
Optical properties reconstruction using the adjoint method based
}

\author{
on the radiative transfer equation
}

\author{
Ahmad Addoum $^{1}$, Olivier Farges ${ }^{1}$, Fatmir Asllanaj $^{1,2 *}$ \\ ${ }^{1}$ Université de Lorraine, LEMTA, UMR 7563, Vandoeuvre-lès-Nancy, France \\ ${ }^{2}$ CNRS, LEMTA, UMR 7563, Vandoeuvre-lès-Nancy, France \\ ${ }^{*}$ Correspondence author - Phone: +33 383595526 ; Fax: +33 383595551 \\ E-mail: Fatmir.Asllanaj@univ-lorraine.fr
}

\begin{abstract}
An efficient algorithm is proposed to reconstruct the spatial distribution of optical properties in heterogeneous media like biological tissues. The light transport through such media is accurately described by the radiative transfer equation in the frequency-domain. The adjoint method is used to efficiently compute the objective function gradient with respect to optical parameters. Numerical tests show that the algorithm is accurate and robust to retrieve simultaneously the absorption $\mu_{a}$ and scattering $\mu_{s}$ coefficients for lowly and highly absorbing medium. Moreover, the simultaneous reconstruction of $\mu_{s}$ and the anisotropy factor $g$ of the Henyey-Greenstein phase function is achieved with a reasonable accuracy. The main novelty in this work is the reconstruction of $g$ which might open the possibility to image this parameter in tissues as an additional contrast agent in optical tomography.
\end{abstract}

Keywords Optical properties; image reconstruction; radiative transfer equation; adjoint method; crosstalk problem; anisotropy factor.

\section{Introduction}

Diffuse Optical Tomography (DOT) is a non-invasive imaging modality which employs a visible or Near infrared Laser source for probing biological tissues and measures light intensities at the boundary surface. In recent years, potential applications of DOT have been developed such as breast cancer detection [1] and brain functional imaging [2]. This tech- 
nique seeks to recover the spatial distributions of optical properties inside the medium through an image reconstruction algorithm. Optical properties are different between healthy and cancerous tissues [3] [4] due to physiological or pathological changes [5]. In spite of all the success of DOT in cancer diagnosis applications, to date, only absorption $\left(\mu_{a}\right)$ and reduced scattering $\left(\mu_{s}^{\prime}\right)$ coefficients reconstruction can be found in the literature. However, the anisotropy factor $g$ of the Henyey-Greenstein (H-G) phase function has an important effect on light propagation [6] and can reveal rich informations on the anisotropic scattering behavior of the tissue: [7] showed that the $g$ value of porcine brain tissue increases from 0.561 to 0.834 after thermal coagulation, [8] demonstrated that the anisotropy factor $g$ of rat liver decreases from 0.952 to 0.946 in a tumor at $633 \mathrm{~nm}$, [9] proved experimentally that $g$ was different for normal human liver tissue and liver metastases at three different wavelengths. That means that $g$ can also be modified when tissue is affected by an eventual tumor besides $\mu_{a}$ and $\mu_{s}$. Therefore, this factor can provide a potential contrast agent for optical medical diagnosis between healthy and tumoral tissues. To our best knowledge, up to now, no works have been done to investigate the reconstruction of the spatial distribution of $g$ [10]. On the other hand, an efficient forward model to predict light transport in the biological tissue is required in order to estimate optical parameters. Many research groups have adopted the diffusion equation as a forward model $[11,12,13]$. However, this model fails to accurately predict light propagation close to sources and boundaries, and also in highly absorbing mediums [14]. An additional drawback is that the estimation of $g$ is not possible ( $g$ is assumed to be constant and known), due to the introduction of the reduced scattering coefficient and then the loss of information about the scattering phase function [6]. To overcome these limitations, more and more interest is turned towards a forward model based on the radiative transfer equation (RTE). The RTE rigorously describes the light propagation in biological tissues. The anisotropy factor $g$ is an independant parameter in the RTE via the H-G phase function. Different forms of the RTE have been used in DOT. The frequency-domain (FD) approach $[15,16,17,18,19,20,21]$ is the most widely employed, since it is a good trade-off between time-domain [22, 23, 24] and steady-state domain [25]. Moreover, the FD approach provides additional information (phase shift) compared to the steady-state modality and avoids the technical limitations of the experimental setup for time-domain often expensive. In addition, the use of FD data allows to better separate the optical properties than the steady-state data by reducing the crosstalk issue when simultaneous estimation is applied [16]. Another challenging task still remains in DOT: the inverse problem. The inversion algorithm can be considered as a large-scale optimization problem, since the optical properties vary spatially inside the medium. In principle, the simultaneous estimation of the three optical properties $\left(\mu_{a}, \mu_{s}\right.$ and $\left.g\right)$ is not possible, due to the non-uniqueness of the ill-posed problem when several optical properties distributions lead to an identical set of boundary data [6]. Additionally, these three parameters differ in nature, units, order of magnitude and sensitivities on the emerging intensity of the forward 
model which makes the estimation inextricable. That's why, in this work, we reconstructed only two parameters either ( $\mu_{a}$ and $\mu_{s}$ ) or $\left(\mu_{s}\right.$ and $g$ ) simultaneously in order to reduce the ill-posed nature of the problem. This inversion aims at recovering the optical properties of tissue through the minimization of an appropriate objective function (OF). Most of the time, the $\mathrm{OF}$ is the least-square error norm between the measured and the predicted data calculated by the forward model. Gradient-based algorithms are commonly used as optimization methods $[13,16,26]$, which employ the gradient of the OF with respect to the optical parameters to find the minimum. These methods proved to be efficient and robust in DOT [24]. On the other side, the core problem and difficulty in the inversion procedure is to accurately compute the gradient of the OF. This process can be computational-intensive due to the number of parameters to retrieve which are space dependent. Generally, the adjoint differentiation is the most commonly used method for calculating the gradient because it uses only elementary results at each iteration step of the forward model [27, 28]. However, when the dimension of the problem is high (larger than 1000 for example), the use of this method becomes cumbersome and computationaly expensive. More recently, [29] employed the adjoint method which gives an efficient and fast way to compute the OF gradient regardless of the number of unknowns. This is done by solving an additionnal (adjoint) equation for the adjoint variable whose computational cost is equivalent to that of the forward calculation.

In this work, a gradient-based algorithm using the RTE as forward model is employed to reconstruct the optical properties $\left(\mu_{a}, \mu_{s}\right.$ and $g$ ) of a heterogeneous medium. The gradient of the OF is obtained accurately by means of the adjoint method in the FD. The objective of this study is to test the efficiency and the robustness of the proposed algorithm in presence of some issues encountered in the DOT. These issues such as the collimated source number, the crosstalk between two optical parameters, the inclusion contrast level, the highly absorbing medium, the measurements noise level and the inclusion location effects are examined through several test cases. Furthermore, for author's best knowledge, the estimation of $g$ and the simultaneous reconstruction of $\mu_{s}$ and $g$ have not been reported yet in the previous works. This explains our motivation to test in particular the feasibility of the present method to reconstruct simultaneously $\mu_{s}$ and $g$ with and without crosstalk. First, the RTE equations are described and the detector predictions on the boundary are given. Second, the adjoint method is presented through a lagrangian formalism for the computation of the OF gradient at multiple modulation frequencies. Finally, to illustrate the performance of the algorithm, single and simultaneous reconstructions of optical properties based on numerical test phantoms are presented in presence of certain issues mentioned above. 


\section{Forward model}

In DOT, the light transport in the biological tissues is a forward model which aims at computing the prediction of the detectors reading once the source and the optical properties of the medium are known. The biological tissue is illuminated by an external collimated Laser beam $\Upsilon\left(\boldsymbol{r}_{\boldsymbol{s}}, \omega_{k}\right)$ at the source position $\boldsymbol{r}_{\boldsymbol{s}}$ on the surface. $\omega_{k}$ is the modulation frequency of the intensity-modulated Laser source. In order to take into account this collimated light, the energy arriving in the medium is separated into two components $\psi=\psi_{c}+\psi_{s}$, respectively the collimated $\psi_{c}$ and scattered radiance $\psi_{s}$. The $\psi_{c}$ radiance is governed by the RTE state equation $\mathcal{R}_{c}$ in the collimated direction $\boldsymbol{\Omega}_{c}$ and is solved analytically.

$$
\mathcal{R}_{c}=\left[\boldsymbol{\Omega}_{c} \cdot \nabla+\left(\frac{i \omega_{k}}{v}+\mu_{t}(\boldsymbol{r})\right)\right] \psi_{c}\left(\boldsymbol{r}, \omega_{k}\right)=0
$$

The velocity of light, $v$, in tissue is the ratio $v=c / n$ of the velocity of light in vacuum and the refractive index of tissue. The total extinction coefficient $\mu_{t}(\boldsymbol{r})$, at position $\boldsymbol{r}$, is the sum of the absorption $\mu_{a}(\boldsymbol{r})$ and the scattering $\mu_{s}(\boldsymbol{r})$ coefficients. The boundary condition for the collimated component $\psi_{c}\left(\boldsymbol{r}, \omega_{k}\right)$ is given by:

$$
\psi_{c}\left(\boldsymbol{r}, \omega_{k}\right)=\Upsilon\left(\boldsymbol{r}_{\boldsymbol{s}}, \omega_{k}\right) \text { for } \boldsymbol{\Omega}_{\boldsymbol{c}} \cdot \boldsymbol{n}<0,
$$

where $\boldsymbol{n}$ is the outward normal unit vector of the boundary. It should be noted that the component $\Upsilon$, in Eq. 2, represents only the transmitted part (no reflexion) of the collimated Laser beam into the medium. The scattered radiance $\psi_{s}\left(\boldsymbol{r}, \boldsymbol{\Omega}, \omega_{k}\right)$ is obtained by solving the RTE state equation $\mathcal{R}_{s}$ in the direction $\Omega$ of the light propagation such as :

$$
\mathcal{R}_{s}=\left[\boldsymbol{\Omega} \cdot \nabla+\left(\frac{i \omega_{k}}{v}+\mu_{t}(\boldsymbol{r})\right)\right] \psi_{s}\left(\boldsymbol{r}, \boldsymbol{\Omega}, \omega_{k}\right)-\mu_{s}(\boldsymbol{r}) \int_{\Omega^{\prime}=2 \pi} p\left(\boldsymbol{\Omega}^{\prime}, \boldsymbol{\Omega}\right) \psi_{s}\left(\boldsymbol{r}, \boldsymbol{\Omega}^{\prime}, \omega_{k}\right) d \Omega^{\prime}-S_{c}\left(\boldsymbol{r}, \boldsymbol{\Omega}, \omega_{k}\right)=0 .
$$

The H-G phase function $p\left(\boldsymbol{\Omega}^{\prime} \cdot \boldsymbol{\Omega}\right)$ is the most widely adopted scattering phase function in biomedical optics and has been used here $[23,30]$. This function, is the probability that photons traveling in direction $\boldsymbol{\Omega}^{\prime}$ scatter into direction $\Omega$. The $\mathrm{H}-\mathrm{G}$ phase function mathematical expression in $2 \mathrm{D}$ is given by:

$$
p\left(\boldsymbol{\Omega}^{\prime} \cdot \boldsymbol{\Omega}\right)=\frac{1}{2 \pi} \frac{1-g^{2}(\boldsymbol{r})}{\left(1+g^{2}(\boldsymbol{r})-2 g(\boldsymbol{r}) \boldsymbol{\Omega}^{\prime} \cdot \boldsymbol{\Omega}\right)} .
$$

The anisotropy factor $g(\boldsymbol{r})$ represents the mean cosine of the angles of the scattered directions $\Omega$ with respect to the incident ones $\Omega^{\prime}$. This factor is spatially dependent in our case for the heterogeneous medium. The source term $S_{c}\left(\boldsymbol{r}, \Omega, \omega_{k}\right)$ in Eq. (3) induced by the scattering of the collimated radiance $\psi_{c}\left(\boldsymbol{r}, \omega_{k}\right)$ is given by:

$$
S_{c}\left(\boldsymbol{r}, \boldsymbol{\Omega}, \omega_{k}\right)=\mu_{s}(\boldsymbol{r}) p\left(\boldsymbol{\Omega}_{\boldsymbol{c}}, \boldsymbol{\Omega}\right) \psi_{c}\left(\boldsymbol{r}, \omega_{k}\right)
$$

Eq. (3) is associated to a semi-transparent boundary condition [31] with Fresnel reflection at the interface (air / biological tissue) due to the refractive index mismatch. The detector prediction $P\left(\boldsymbol{r}_{\boldsymbol{d}}, \omega_{k}\right)$ corresponding to the exitance at the 
detector position $\boldsymbol{r}_{\boldsymbol{d}}$ on the illuminated surface is obtained by:

$$
P\left(\boldsymbol{r}_{\boldsymbol{d}}, \omega_{k}\right)=\int_{\boldsymbol{n} \cdot \boldsymbol{\Omega}^{\prime}>0}[1-\rho(\Theta)] \psi_{s}\left(\boldsymbol{r}, \boldsymbol{\Omega}^{\prime}, \omega_{k}\right)\left(\boldsymbol{\Omega}^{\prime} \cdot \boldsymbol{n}\right) d \Omega^{\prime},
$$

where $\rho(\Theta)$ is the reflectivity of the surface $\partial \mathcal{D}$. The forward model has been solved accurately with a Modified Finite Volume Method (MFVM). The methodology of this method is not repeated here and we refer the reader to [32], for details. The stability and accuracy of the MFVM have been validated through comparisons with the Monte Carlo (MC) technique and analytical solution of RTE on available test cases. The MFVM, compared to other deterministic numerical solutions of the RTE (available in the literature) has the advantage to have a high precision with an error less than $1 \%$ with respect to MC simulations and RTE analytical solution. This is mainly due to the fact that the RTE is also solved inside each control volume through an exponential schema.

\section{Inverse problem}

In the following, we first define the discrete sum of the objective function over all modulation frequencies. Then we introduce the minimization problem where the state equations are used as constraints. After introducing the Lagrangian formalism, we show how to deduce the adjoint equations and the objective function gradient.

\subsection{Objective function and minimization problem}

The $\mathrm{OF} \mathcal{J}(\beta)$ to be minimized in the inversion procedure is the mean square discrepancy between the measurements, $M\left(\boldsymbol{r}_{\boldsymbol{d}}, \omega_{k}\right)$, and the predictions of the forward model based on the RTE, $P\left(\boldsymbol{r}_{\boldsymbol{d}}, \omega_{k}\right)$, at detector positions, $\boldsymbol{r}_{\boldsymbol{d}}$ on the surface of the medium over all intensity modulation frequencies $\omega_{k}$ :

$$
\mathcal{J}(\beta)=\frac{1}{2} \sum_{\omega_{k=1}}^{N_{\omega}} J\left(\beta, \omega_{k}\right) \quad \text { with } \quad J\left(\beta, \omega_{k}\right)=\sum_{d=1}^{N_{d}}\left\|P\left(\boldsymbol{r}_{\boldsymbol{d}}, \omega_{k}\right)-M\left(\boldsymbol{r}_{\boldsymbol{d}}, \omega_{k}\right)\right\|^{2},
$$

The vector $\beta$ contains the spatial distribution of the optical properties in the heterogeneous medium. Here $N_{d}$ and $N_{\omega}$ are the total numbers of detectors and modulation frequencies, respectively. The goal of the optimization technique is to

determine the vector $\hat{\beta}$ that minimizes the OF iteratively. This vector $\hat{\beta}$ will be a solution to the minimization problem and is displayed as a two-dimensional optical image.

\subsection{Lagrangian and adjoint model equations}

The adjoint equations are derived by considering that the OF at each modulation frequency $J\left(\beta, \omega_{k}\right)$ must be minimized under some constraints given by the RTE state equations at the corresponding frequency $\omega_{k}$. Hence, we can rewrite the 
original OF by following the Lagrangian formalism of the minimization problem as:

$$
\mathcal{L}\left(\beta, \psi_{s}, \psi_{s}, \lambda_{s}, \lambda_{c}\right)=\sum_{\omega_{k=1}}^{N_{\omega}}\left[J\left(\beta, \omega_{k}\right)+\left(\lambda_{s} \mid \mathcal{R}_{s}\left(\beta, \psi_{s}, \psi_{c}\right)\right)_{s}^{\omega_{k}}+\left(\lambda_{c} \mid \mathcal{R}_{c}\left(\beta, \psi_{c}\right)\right)_{c}^{\omega_{k}}\right]
$$

where $\lambda_{c}=\lambda_{c}\left(\boldsymbol{r}, \omega_{k}\right), \lambda_{s}=\lambda_{s}\left(\boldsymbol{r}, \Omega, \omega_{k}\right)$ are the complex adjoint variables to $\psi_{c}$ and $\psi_{s}$, respectively. The inner products $(\cdot \mid \cdot)_{c}^{\omega_{k}}$ and $(\cdot \mid \cdot)_{s}^{\omega_{k}}$ associated to the solution space, respectively, of $\psi_{c}$ and $\psi_{s}$ are defined by:

$$
\begin{gathered}
\left(\lambda_{s} \mid \mathcal{R}_{s}\right)_{s}^{\omega_{k}}=\mathbf{R e} \int_{\mathcal{D}} \int_{\Omega=2 \pi} \overline{\lambda_{s}\left(\boldsymbol{r}, \boldsymbol{\Omega}, \omega_{k}\right)} \mathcal{R}_{s}\left(\beta, \psi_{s}, \psi_{c}\right) d \Omega d r \\
\left(\lambda_{c} \mid \mathcal{R}_{c}\right)_{c}^{\omega_{k}}=\mathbf{R e} \int_{\mathcal{D}} \overline{\lambda_{c}\left(\boldsymbol{r}, \omega_{k}\right)} \mathcal{R}_{c}\left(\beta, \psi_{c}\right) d r .
\end{gathered}
$$

When $\psi_{c}, \psi_{s}$ verify the state equations Eqs. (1), (3), respectively, that leads to:

$$
\mathcal{L}\left(\beta, \psi_{s}, \psi_{c}, \lambda_{s}, \lambda_{c}\right)=\mathcal{J}(\beta), \quad \mathcal{L}^{\prime}\left(\beta, \psi_{s}, \psi_{c}, \lambda_{s}, \lambda_{c}\right)=\mathcal{J}^{\prime}(\beta)
$$

Using Eq. (11), we can extract the gradient of the OF from the $L_{2}$ inner product of the directional differential $\mathcal{L}^{\prime}$ with respect to $\beta$ in the direction $\delta \beta$, such that:

$$
\mathcal{L}^{\prime}(\beta)=(\nabla \mathcal{J}(\beta) \mid \delta \beta)_{L_{2}}
$$

Notice that the functional $\mathcal{L}$ is independant of $\lambda_{s}$ and $\lambda_{c}$ due to the fact that the residuals $\mathcal{R}_{s}$ and $\mathcal{R}_{c}$ are zero, yielding:

$$
\frac{\partial \mathcal{L}\left(\beta, \psi_{s}, \psi_{c}\right)}{\partial \lambda_{s}}=0, \quad \frac{\partial \mathcal{L}\left(\beta, \psi_{s}, \psi_{c}\right)}{\partial \lambda_{c}}=0
$$

The lagrangian formalism assumes that the variation of $\partial \mathcal{L}$ is not non-zero unless there is a variation of $\beta$. This condition is ensured by a particular choice of adjoint variables $\lambda_{s}$ and $\lambda_{c}$ which allows to compute the OF gradient without having to compute the sensitivities $\delta \psi_{s}=\left(\partial \psi_{s}\left(\boldsymbol{r}, \boldsymbol{\Omega}, \omega_{k}\right) / \partial \beta\right) \delta \beta$ and $\delta \psi_{c}=\left(\partial \psi_{c}\left(\boldsymbol{r}, \omega_{k}\right) / \partial \beta\right) \delta \beta$. These sensitivities are computationally expensive. Hence, that leads to the following adjoint equations model of the FD DOT problem:

$$
\partial_{\psi_{s}} \mathcal{L}\left(\beta, \psi_{s}, \psi_{c}\right) \delta \psi_{s}=0, \quad \partial_{\psi_{c}} \mathcal{L}\left(\beta, \psi_{s}, \psi_{c}\right) \delta \psi_{c}=0
$$

The adjoint equations can be obtained by partially differentiating the Lagrangian functional Eq. (8) with respect to $\psi_{s}$, $\psi_{c}$ in direction $\delta \psi_{s}$ and $\delta \psi_{c}$, respectively. After using the definition of the adjoint operator [33] and the inner products properties, we can reformulate the adjoint equations over $N_{\omega}$ modulation frequencies $\omega_{k}$ such as:

$$
\begin{gathered}
\partial_{\psi_{s}} \mathcal{L}\left(\beta, \psi_{s}, \psi_{c}\right) \delta \psi_{s}=\sum_{\omega_{k=1}}^{N_{\omega}}\left[\frac{\partial J\left(\beta, \omega_{k}\right)}{\partial \psi_{s}}+\left(\frac{\partial \mathcal{R}_{s}\left(\beta, \psi_{s}, \psi_{c}\right)}{\partial \psi_{s}}\right)^{*} \lambda_{s}\right]=0, \\
\partial_{\psi_{c}} \mathcal{L}\left(\beta, \psi_{s}, \psi_{c}\right) \delta \psi_{c}=\sum_{\omega_{k=1}}^{N_{\omega}}\left[\left(\frac{\partial \mathcal{R}_{s}\left(\beta, \psi_{s}, \psi_{c}\right)}{\partial \psi_{c}}\right)^{*} \lambda_{s}+\left(\frac{\partial \mathcal{R}_{c}\left(\beta, \psi_{c}\right)}{\partial \psi_{c}}\right)^{*} \lambda_{c}\right]=0 .
\end{gathered}
$$

Note that $\partial J\left(\beta, \omega_{k}\right) / \partial \psi_{c}=0$, since the OF is independent on the collimated light source. 
By using the definitions in Eq. (1) and (3) for $\mathcal{R}_{c}$ and $\mathcal{R}_{s}$, respectively, the adjoint variables must be a solution of the following adjoint equations system at each modulation frequency $\omega_{k}$ :

$$
\begin{gathered}
{\left[-\boldsymbol{\Omega} \cdot \nabla+\left(\frac{-i \omega_{k}}{v}+\mu_{t}(\boldsymbol{r})\right)\right] \lambda_{s}\left(\boldsymbol{r}, \boldsymbol{\Omega}, \omega_{k}\right)-\mu_{s}(\boldsymbol{r}) \int_{\Omega^{\prime}=2 \pi} p\left(\boldsymbol{\Omega}^{\prime}, \boldsymbol{\Omega}\right) \lambda_{s}\left(\boldsymbol{r}, \boldsymbol{\Omega}^{\prime}, \omega_{k}\right) d \boldsymbol{\Omega}^{\prime}+\frac{\partial J\left(\beta, \omega_{k}\right)}{\partial \psi_{s}}=0} \\
{\left[-\boldsymbol{\Omega}_{c} \cdot \nabla+\left(\frac{-i \omega_{k}}{v}+\mu_{t}(\boldsymbol{r})\right)\right] \lambda_{c}\left(\boldsymbol{r}, \omega_{k}\right)-\mu_{s}(\boldsymbol{r}) \int_{\Omega=2 \pi} p\left(\boldsymbol{\Omega}_{\boldsymbol{c}}, \boldsymbol{\Omega}\right) \lambda_{s}\left(\boldsymbol{r}, \boldsymbol{\Omega}, \omega_{k}\right) d \boldsymbol{\Omega}=0}
\end{gathered}
$$

The system of the RTE adjoint equations can be solved with the same solution method as the forward model equations. In the next section, we will show how to obtain the gradient vector components $\nabla \mathcal{J}$ with respect to the optical properties ( $\mu_{a}, \mu_{s}$ and $g$ ) by simply using the adjoint variables $\lambda_{s}\left(\boldsymbol{r}, \Omega, \omega_{k}\right)$ and $\lambda_{c}\left(\boldsymbol{r}, \omega_{k}\right)$ of the adjoint model described above.

\subsection{Gradient expressions}

The directional derivative of the objective function $\mathcal{J}^{\prime}$ is equal to that of the lagrangian functional $\mathcal{L}^{\prime}$ (see Eq. (11)). Thus, it suffices to determine the latter in order to extract the gradient $\nabla \mathcal{J}$. By applying Eqs. (11 - 14), the gradient $\nabla \mathcal{J}$ can be deduced by differentating the lagrangian functional $\mathcal{L}$ with respect to $\beta$ in direction $\delta \beta$ such as:

$$
(\nabla \mathcal{J}(\beta) \mid \delta \beta)_{L_{2}}=\sum_{\omega_{k=1}}^{N_{\omega}}\left[\left(\lambda_{c} \mid \frac{\partial \mathcal{R}_{c}\left(\beta, \psi_{c}\right)}{\partial \beta} \delta \beta\right)_{c}^{\omega_{k}}+\left(\lambda_{s} \mid \frac{\partial \mathcal{R}_{s}\left(\beta, \psi_{s}, \psi_{c}\right)}{\partial \beta} \delta \beta\right)_{s}^{\omega_{k}}\right] .
$$

Note that $\partial J\left(\beta, \omega_{k}\right) / \partial \beta=0$ as the OF does not depend explicitly on $\beta$ (see Eq.(7)).

This latter expression clearly shows that only a simple inner product has to be calculated. It should be noted that, the gradient of the OF at multiple frequencies is the sum of all the gradients computed at each modulation frequency $\omega_{k}$.

$$
(\nabla \mathcal{J}(\beta) \mid \delta \beta)_{L_{2}}=\sum_{\omega_{k=1}}^{N_{\omega}}\left(\nabla J\left(\beta, \omega_{k}\right) \mid \delta \beta\right)_{L_{2}}
$$

Applying Eq. (19) for $\delta \mu_{a}, \delta \mu_{s}$ and $\delta g$, we obtain an analytical expression of the OF gradient, with respect to $\mu_{a}, \mu_{s}$ and $g$, respectively:

$$
\begin{gathered}
\left(\nabla \mathcal{J}\left(\mu_{a}\right) \mid \delta \mu_{a}\right)_{L_{2}}=\sum_{\omega_{k=1}}^{N_{\omega}}\left[\left(\lambda_{s} \mid \psi_{s} \delta \mu_{a}\right)_{s}^{\omega_{k}}+\left(\lambda_{c} \mid \psi_{c} \delta \mu_{a}\right)_{c}^{\omega_{k}}\right] \\
\left(\nabla \mathcal{J}\left(\mu_{s}\right) \mid \delta \mu_{s}\right)_{L_{2}}=\sum_{\omega_{k=1}}^{N_{\omega}}\left[\left(\lambda_{s} \mid \psi_{s} \delta \mu_{s}\right)_{s}^{\omega_{k}}+\left(\lambda_{c} \mid \psi_{c} \delta \mu_{s}\right)_{c}^{\omega_{k}}\right. \\
\left.-\left(\lambda_{s} \mid\left(\int_{\Omega^{\prime}=2 \pi} \psi_{s} p\left(\boldsymbol{\Omega}^{\prime}, \boldsymbol{\Omega}\right) d \Omega^{\prime}+\psi_{c} p\left(\boldsymbol{\Omega}_{c}, \boldsymbol{\Omega}_{k}\right)\right) \delta \mu_{s}\right)_{s}^{\omega_{k}}\right] \\
(\nabla \mathcal{J}(g) \mid \delta g)_{L_{2}}=\sum_{\omega_{k=1}}^{N_{\omega}}\left(\lambda_{s} \mid-\mu_{s}\left(\int_{\Omega^{\prime}=2 \pi} \psi_{s} \frac{\partial p\left(\boldsymbol{\Omega}^{\prime}, \boldsymbol{\Omega}\right)}{\partial g} d \Omega^{\prime}+\psi_{c} \frac{\partial p\left(\boldsymbol{\Omega}_{c}, \boldsymbol{\Omega}\right)}{\partial g}\right) \delta g\right)_{s}^{\omega_{k}}
\end{gathered}
$$

The gradient is then computed for all the optical parameters regardless of the number of unknowns of the problem. Thus, the adjoint formulation gives a fast way to efficiently compute the gradient. This is done by solving an additional 
equation for the adjoint variables and then evaluating the gradient through a simple inner product. To our knowledge, the gradient expression $\nabla \mathcal{J}(g)$ with respect to $g$ is derived for the first time by using the adjoint model of the RTE. Once the gradient $\nabla \mathcal{J}(\beta)$ is obtained accurately, a reconstruction scheme based on the Limited memory (Lm) BFGS method [29] is employed in order to update the spatial distribution of the optical properties. The advantage of this method is that it avoids the cost of building the Hessian and inverting it as in the Newton methods [34, 35]. After computing the Lm-BFGS search direction, the Armijo line search [36] is employed to find an optimal step size, $\alpha^{k}$, which minimises sufficiently the OF.

\section{Results and discussions}

\subsection{Model description}

The reconstruction of the spatial distribution of optical properties in a non-homogeneous biological medium is studied. A two-dimensional $4 \times 4 \mathrm{~mm}^{2}$ domain which contains three circular inclusions is examined as shown in Fig. 1(a). The inclusions $(0.5 \mathrm{~mm}$ in diameter each, centered at $x=-0.1 \mathrm{~mm} ; y=1 \mathrm{~mm}$ for inclusion $\mathrm{A}, x=1 \mathrm{~mm} ; y=1 \mathrm{~mm}$ for inclusion B and $x=-1 \mathrm{~mm} ; y=-1 \mathrm{~mm}$ for inclusion C) are embedded as heterogeneities in the background medium.

[Figure 1 about here.]

$\mathrm{w}<$ The optical properties of these inclusions can take different values for the different test cases considered below. The refractive index of the medium is uniformly set at $n=1.4$ while that of the surrounding medium (air) is set to unity. For all these cases, the homogeneous background optical properties are used as the initial guesses to start the inverse procedure. Four Gaussian Laser sources illuminate simultaneously the mid-center of each side of the medium, unless the cases specified otherwise (those using one source). The expression of the Gaussian function in space along $x$-axis or $y$ -axis $(s=x$ or $y)$ is given by:

$$
\Upsilon(s)=\frac{1}{\sigma_{s} \sqrt{2 \pi}} \exp \left(-\frac{s^{2}}{2 \sigma_{s}^{2}}\right),
$$

where $\sigma_{s}=0.5 \mathrm{~mm}$ is the standard deviation of the spatial Gaussian beam. Eighty detectors are distributed around the numerical phantom (one source and 20 detectors are located on each side of the medium). All detectors predictions were used in the minimization problem, expect the cases where $\mu_{s}$ and $g$ factor are reconstructed simultaneously. The synthetic data were obtained by running the forward model using the exact heterogeneous distribution of the optical properties we want to reconstruct. An unstructured triangular mesh of $N_{s}=2577$ nodes (degrees of freedom) corresponding to 4992 triangles was used (Fig. 1(b)). The angular space was discretized into 32 directions and each direction was also subdivided 
into 8 solid angles for the normalized phase function. The reconstructions were achieved by fitting the FD data obtained at 10 modulation frequencies equally distributed in the range of $100 \mathrm{MHz}$ to $1 \mathrm{GHz}$. We have found that the multifrequency approach provides a better estimation quality than that at single modulation frequency $[37,38,39,40]$. This is because the underdetermination of the large-scale optimization problem was reduced by employing multiple modulation frequencies. The reconstruction process is terminated when the normalized difference of the OF between two subsequent iteration steps was smaller than $\epsilon=10^{-4}$. To compare the quality of reconstruction images, the relative error $\varepsilon$ between real and reconstructed values of optical properties is defined as:

$$
\varepsilon_{\beta}(Z I)=\frac{100}{P} \sum_{i=1}^{Z I}\left\|\frac{\hat{\beta}_{i}-\beta_{i}^{*}}{\beta_{i}^{*}}\right\|,
$$

where $\hat{\beta}_{i}$ and $\beta_{i}^{*}$ are the reconstructed and the exact values of the optical parameter at the $i^{\text {th }}$ node of the mesh, respectively. $P$ represents the unknowns number (mesh nodes) in the zone of interest $Z I$ of the image. This $Z I$ can be either the background, inclusion or cross-talk zone or even the whole reconstructed image.

\subsection{Impact of source number on reconstruction}

As the first test problem, the effect of source number on reconstruction is examined. For this purpose, we consider the phantom described above where only the scattering coefficient varies spatially inside the medium through the inclusions. The optical properties of the background are chosen in the range of biological tissues $\left(\mu_{a}=0.05 \mathrm{~mm}^{-1}, \mu_{s}=5 \mathrm{~mm}^{-1}\right.$ and $g=0.9$ ). These properties yield to a highly-forward anisotropically scattering medium. Inclusions $\mathrm{A}$ and $\mathrm{C}$ are assigned the real scattering coefficient $\mu_{s}^{*}=4 \mathrm{~mm}^{-1}$, while inclusion B is assigned the real $\mu_{s}^{*}=6 \mathrm{~mm}^{-1}$, corresponding to a $20 \%$ decrease and increase, respectively, relative to the scattering coefficient of the background medium. Figs. 2(a) and 2(b) display the reconstructed images of $\mu_{s}$ when only the top surface of the medium was probed and when the four different boundaries of the medium were illuminated, respectively.

[Figure 2 about here.]

From Figs. 2(a,b), it can be seen that the three inclusions are spatially well recovered in the exact locations for both cases. As expected, the two top inclusions (A and B) are accurately reconstructed while the deeper one (inclusion C) is significantly overestimated $\left(\hat{\mu_{s}}=4.5 \mathrm{~mm}^{-1}\right)$ when probing only the north boundary. In addition, the inclusion $\mathrm{C}$ has worse contrast and circular shape (Fig. 2(a)). However, the circular shape and the contrast of the inclusion C are clearly improved and enhanced when scaning the medium from all its boundaries. The scattering values are accurately estimated $\hat{\mu_{s}}=4 \mathrm{~mm}^{-1}, \hat{\mu_{s}}=6 \mathrm{~mm}^{-1}$ and $\hat{\mu_{s}}=4 \mathrm{~mm}^{-1}$ at the centers of inclusions A, B and C, respectively (Fig. 2(b)). 


\subsection{Simultaneous reconstruction of $\mu_{a}$ and $\mu_{s}$}

In this section, the simultaneous reconstruction of the spatially dependent absorption and scattering distribution is examined with three different test cases. The anisotropy factor of the H-G phase function is kept constant $g=0.9$. We reconsider the same phantom as described in Fig. 1, illuminated by four collimated Laser beams.

The most encountered issues and challenges in DOT are reported in this section in order to assess the robustness of the reconstruction algorithm. Firstly, the "crosstalk" problem is frequently encountered in practice when simultaneous estimation of several parameters is applied. This problem is due to the non-uniqueness of the DOT where many combinations of optical properties can lead to similar boundary data. For this purpose, we assumed that the inclusion A represents a heterogeneity with low in both absorption and scattering coefficients compared to the background optical properties. Whereas, inclusion $\mathrm{B}$ is highly scattering only while inclusion $\mathrm{C}$ is highly absorbing only. This configuration is intended to mimic a crosstalk in the three test media. Secondly, the "contrast" level represents the difference between the background (initial optical value) and the inclusion (exact optical value). Different contrasts may lead to different reconstruction results $[41,42]$. In the 3 test cases, the three inclusions represent a contrast of $20 \%$ with respect to the background optical values expect for case 2 where inclusions B and C represent a contrast of $40 \%$. This case is chosen in order to evaluate the effect of this contrast on the crosstalk issue and the estimation quality. Two homogeneous backgrounds of different optical properties are employed. The first two cases are assigned the low-absorbing background medium of $\left(\mu_{a}=0.05 \mathrm{~mm}^{-1}, \mu_{s}=5 \mathrm{~mm}^{-1}\right)$ while the third one consists of a very high-absorbing medium $\left(\mu_{a}=1 \mathrm{~mm}^{-1}, \mu_{s}=5 \mathrm{~mm}^{-1}\right)$. This last case presents a situation in which the diffusion approximation is not valid. The exact optical properties of the inclusions for each case are listed in Table 1. Figure 3 displays the reconstructed $\mu_{a}$ and $\mu_{s}$ images for the 3 test mediums. The relative reconstruction errors of background, inclusions and crosstalk are given in Table 2 .

[Table 1 about here.]

[Figure 3 about here.]

As shown in Fig. 3, the inclusions are accurately located in both optical parameters for all test cases. For the two low-absorbing media, perturbations such as edges artifacts are more remarkable in the absorption images. Also, the local values in the scattering maps are accurately retrieved while that of the absorption maps are underestimated. The purely absorbing inclusion $\mathrm{C}$ has no crosstalk impact on the $\mu_{s}$ images even with hight contrast of $40 \%$ (see Figs. 3(b,d)). This is because the use of the FD data is expected to better separate between the two parameters. However, the crosstalk 
phenomenon is only pronounced in absorption images. As shown in Figs. 3(a,c), the scattering inclusion B appeared as a false positive heterogeneity in the $\mu_{a}$ images for both cases. This behavior is mainly due to the different sensitivities of the RTE model which is much more sensitive to variations in $\mu_{s}$ than $\mu_{a}$ [43]. These parameters have somehow the same effect on the boundary data. In other words, a decrease in intensity could be caused by either an increase in absorption or in scattering [6], since both contribute to light extinction in tissue. Therefore, the low emerging intensities caused by the highly scattering inclusion B, have been analyzed as a highly inclusion in both scattering and absorption (crosstalk).

By comparing the cases 1 and 2, we find that the estimation errors of the inclusions B and C with $20 \%$ of contrast, $\varepsilon_{\mu_{s}}^{I n c B}$ $=5.46 \%$ and $\varepsilon_{\mu_{a}}^{I n c C}=9.16 \%$, have been approximately doubled to $\varepsilon_{\mu_{s}}^{I n c B}=9.70 \%$ and $\varepsilon_{\mu_{a}}^{I n c C}=17.7 \%$, respectively, when the contrast level was increased about $40 \%$ (see Table 2). However, the inclusion A error was slightly increased to $\varepsilon_{\mu_{a}}^{I n c A}$ $=10.95 \%$ and $\varepsilon_{\mu_{s}}^{I n c A}=7.35 \%$ because the contrast level for this inclusion has not changed for the two cases. In the case 2 , perturbations are more pronounced in the absorption background $\left(\varepsilon_{\mu_{a}}^{\text {Background }}=6.11 \%\right)$ and some artifacts are also remarkable in the scattering image $\left(\varepsilon_{\mu_{s}}^{\text {Background }}=1.36 \%\right)$. Thus, it is interesting to note that the high inclusion contrast leads to more artifacts around the boundary while the low inclusion contrast shows better estimation quality. As shown in Figs. 3(a,c), the reconstructed absorbing inclusion $\mathrm{C}$ is readily visible when the contrast is up to $40 \%$. This is because the influence of the last becomes more important on the emerging data with higher contrast. Furthermore, it is also seen that the contrast has some considerable effect on the crosstalk. The crosstalk error in the absorption map $\varepsilon_{\mu_{a}}^{\text {Crosstalk }}=$ $12.29 \%$ has been doubled to $\varepsilon_{\mu_{a}}^{\text {Crosstalk }}=24.49 \%$ when the contrast of the inclusion B was increased from $20 \%$ to $40 \%$, respectively. For the scattering map the crosstalk error $\varepsilon_{\mu_{s}}^{C r o s s t a l k}=0.17 \%$ was slightly increased to $\varepsilon_{\mu_{s}}^{C r o s s t a l k}=0.27 \%$, but remains low. Hence, a large contrast level leads to a worse estimation quality and further to a strong crosstalk effect in the absorption images. This has an important implication because the realistic contrast levels between tumor and normal tissue are believed to be in the range of the lower contrast levels [42].

For the test case 3, the reconstruction of optical properties is achieved with a reasonable accuracy thanks to the RTE based forward model. This result would not have been possible with the diffusion approximation, since it fails to predict accurately the light propagation in such medium. Comparing now the tests 1 and 3 , we can observe that the reconstruction of the $\mu_{a}$ background becomes clearer with less perturbations leading to lower relative error of $\varepsilon_{\mu_{a}}^{\text {Background }}=0.94 \%$ in the highly-absorbing medium (Figs. 3(a,e)). In addition, the crosstalk error induced by the scattering inclusion B has been reduced about $50 \%\left(\varepsilon_{\mu_{a}}^{\text {Crosstalk }}=5.769 \%\right)$ in the $\mu_{a}$ image. The estimation errors of the inclusions $\mathrm{A}$ and $\mathrm{C}\left(\varepsilon_{\mu_{a}}^{I n c A}=\right.$ $11.62 \%, \varepsilon_{\mu_{a}}^{I n c C}=9.94 \%$ ) are somewhat similars compared to errors obtained in case 1. This is because the contrast level of $20 \%$ was kept unchanged in the two test cases. For the $\mu_{s}$ image, the background is accurately recovered with small 
error of $\varepsilon_{\mu_{s}}^{\text {Background }}=0.60 \%$. In contrary to case 1 and 2 , the purely absorbing inclusion $\mathrm{C}$ has now a significant crosstalk effect on the $\mu_{s}$ image (Figs. 3(b,f)). Nevertheless, the crosstalk error is still relatively small $\left(\varepsilon_{\mu_{s}}^{\text {Crosstalk }}=1.85 \%\right)$ in the $\mu_{s}$ image. The estimation errors of the inclusions $\mathrm{A}$ and $\mathrm{B}$ were increased to almost $50 \% \varepsilon_{\mu_{s}}^{I n c A}=12.1 \%$ and $\varepsilon_{\mu_{s}}^{I n c B}$ $=12.6 \%$ compared to the case 1 , respectively. One can deduce here that the reconstruction quality of the $\mu_{a}$ image is improved (low crosstalk and background errors) while that of the $\mu_{s}$ image is worse (high crosstalk and inclusions errors). This improvement of $\mu_{a}$ can be explained by the highly absorption coefficient yielding to a comparable order of magnitude with the scattering coefficient. In this situation, the RTE model has a significant sensitivity to any small variation of $\mu_{a}$ (20\%) when the absorption coefficient is very high. Therefore, the algorithm provides a better reconstruction quality for $\mu_{a}$. On the other hand, the highly absorbing coefficient produces a high light extinction in the tissue which attenuates the multiple scattering of light and therefore to $\mu_{s}$ inaccuracy.

On the other hand, if the $\mu_{s}$ and $\mu_{a}$ original images contain each two inclusions where their exact values are both either high or low with respect to their initial values, the proposed algorithm was tested and has been proven to reconstruct accurately this example case.

[Table 2 about here.]

\subsection{Reconstruction of $g$}

In this case, the anisotropy factor $g$ varies spatially inside the medium through the inclusions as represented in Fig. 1(a). The algorithm was used to reconstruct the spatial distribution of $g$ when $\mu_{a}$ and $\mu_{s}$ are assumed to be known. The optical properties of the homogeneous background are $\mu_{a}=0.05 \mathrm{~mm}^{-1}, \mu_{s}=5 \mathrm{~mm}^{-1}$ and $g=0.9$. The inclusions A and C are assigned the exact value $\mathrm{g}^{*}=0.85$, while the inclusion $\mathrm{B}$ is assumed to have higher forward-scattering value $\mathrm{g}^{*}=0.95$. In order to test the robustness of the algorithm, the synthetic data are corrupted by adding normal distributed random errors to the exact predictions such as:

$$
M_{d}=P_{d}^{*}+\operatorname{rand} \sigma_{d}
$$

where $M_{d}$ and $P_{d}^{*}$ are the measured value and the exact prediction at the $d$-th detector on the bounding surface, respectively. The rand function, in Eq. (26), generates random numbers with a normal Gaussian distribution. The noise level $\sigma_{d}$ is defined as the standard deviation of the measured value at the $d$-th detector. Four examples of different noise levels present in the synthetic data $(0 \%, 3 \%, 6 \%$ and $10 \%)$ were used in the reconstructions. The results are depicted in Figure 4 and the computational features of the reconstruction algorithm are given in Table 3.

[Figure 4 about here.] 
For the noise-free example, the reconstructed g image is in good agreement with the original medium (Fig. 4(a)). Locations and circular shapes of the inclusions were clearly reconstructed. The retrieved local values at the inclusions centers were accurately estimated leading to low relative error of the inverted image $\varepsilon_{g}=0.29 \%$. Also, the background anisotropy factor is well recovered for all the cases. These results show that the gradient expression with respect to the anisotropy factor (Eq. 23) has been validated and accurately computed by the present adjoint method. For the noisy data examples, it is also seen that the algorithm can well detect and locate the inclusions inside the medium (Figs. 4(b, c, d)). However, the edges artifacts are more pronounced and the circular shape of inclusions is distorted as the noise level in found to increase. Additionally, the estimation error increases to $\varepsilon_{g}=0.41 \%, \varepsilon_{g}=0.53 \%$ and $\varepsilon_{g}=0.72 \%$ when the noise level increases to $3 \%, 6 \%$ and 10\%, respectively (see Tab. 3). As expected, higher noise levels on the boundary data lead to quality image degradation. From Table 3, the optimization procedure reached the stopping criterion faster for a higher noise level. This is because the OF converges around a certain noisy value. This computational feature is often encountered for gradient based algorithms and has been reported in $[10,36]$. It should be noted here that the estimation of $g$ would have not been possible with the diffusion approximation. Since, the information about the anisotropy factor is lost by considering the reduced scattering coefficient $\mu_{s}^{\prime}=\mu_{s}(1-g)$ in the diffusion equation.

[Table 3 about here.]

\subsection{Simultaneous reconstruction of $\mu_{s}$ and $g$}

\subsubsection{With crosstalk problem}

In this section, the spatial distributions of the scattering coefficient $\mu_{s}$ and the anisotropy factor $g$ are reconstructed simultaneously. The original phantom to reconstruct contains only the two top inclusions A and B located as depicted in Fig. 1(a). The medium was probed by one Gaussian source at the mid-center of the top surface and only the backscattered light (reflectance) on the illuminated boundary was used for reconstruction. The use of one source allows to highlight the inclusion location effect with respect to the source on the estimation and crosstalk qualities. In this source-detectors configuration, we are able to assess the sensitivities of the $\mu_{s}$ coefficient and the $g$ factor on the reflectance. The homogeneous background parameters are the same as in the previous section. For author's best knowledge, to date, the crosstalk problem between $\mu_{s}$ and $g$ has not been considered in the literature for the DOT. Hence, we are interested to study the crosstalk effect of the scattering coefficient on the anisotropy factor reconstruction, and vice-versa. For this purpose, two different test cases are considered. In the first one, the inclusion A varies only in scattering $\left(\mu_{s}^{*}=4 \mathrm{~mm}^{-1}\right) \mathrm{while}^{\mathrm{s}}$ the inclusion B represents a heterogeneity in anisotropy factor $\left(g^{*}=0.85\right)$. In the second test, the inclusion A is an anisotropy 
heterogeneity only $\left(g^{*}=0.85\right)$ and the inclusion B varies only in scattering $\left(\mu_{s}^{*}=4 \mathrm{~mm}^{-1}\right)$. This last is made in order to show the influence of the inclusion location with respect to the source on the reconstruction results. The reconstructed images are displayed in Fig. 5 and the relative errors are listed in Table 4.

\section{[Figure 5 about here.]}

From Table 4 , the reconstruction quality of the inclusion $\mathrm{A}$ is relatively more accurate $\left(\varepsilon_{\mu_{s}}^{I n c A}=17.20 \%\right.$ and $\varepsilon_{g}^{I n c A}=$ $3.00 \%)$ than that of the inclusion $\mathrm{B}\left(\varepsilon_{\mu_{s}}^{I n c B}=19.00 \%\right.$ and $\left.\varepsilon_{g}^{I n c B}=4.35 \%\right)$ in scattering and anisotropy factor. This is mainly due to the location effect, since the inclusion $\mathrm{A}$ is nearer to the source and placed in higher sensitivity area than the inclusion B. Therefore, the inclusion A transmits more rich informations to detectors and makes the inversion more accurate. Unlike the cases where $\mu_{a}$ and $\mu_{s}$ were retreived simultaneously, the crosstalk issue between the scattering and the anisotropy factor is clearly pronounced in both optical images. This is because $\mu_{s}$ and $g$ have both significative and important sensitivities on the emerging intensities. Comparing the two test cases, it can be observed that the crosstalk impact depends on the inclusion location with respect to the source. The crosstalk $\mu_{s}$ error has decreased from $\varepsilon_{\mu_{s}}^{C r o s s t a l k}$ $=9.66 \%$ to $\varepsilon_{\mu_{s}}^{\text {Crosstalk }}=8.62 \%$ when the $g$ heterogeneity was exchanged from inclusion A to B. Similarly, the crosstalk $g$ error has decreased from $\varepsilon_{g}^{\text {Crosstalk }}=1.03 \%$ to $\varepsilon_{g}^{\text {Crosstalk }}=0.49 \%$ when the scattering inclusion passed from position A to B. Thus, we can deduce that the crosstalk is more pronounced as the responsible inclusion in the other parameter is nearer to the source (inclusion A). Note that, the $\mu_{s}$ inclusion B (case 2) has a weak crosstalk effect on the $g$ image (Fig. 5d) whereas when this inclusion is a $g$ heterogeneity (case 1), the crosstalk in the $\mu_{s}$ image is much more remarkable (Fig. 5a). This can be attributed to the different sensitivities for $\mu_{s}$ and $g$ on the reflectance. It has been shown in [43] that the reflectance of the RTE model is much more sensitive to a variation in the anisotropy factor than the scattering coefficient. That explains the better estimation quality of $g$ for all cases (Tab. 4) and further the strong crosstalk in the reconstructed $\mu_{s}$ images. Another interesting remark can be observed when $\mu_{s}$ and $g$ are reconstruct simultaneously. As shown in Figs. 5(a,b,c,d), the low inclusion in anisotropy factor appears as a high scattering inclusion in the $\mu_{s}$ images. Also, the low scattering inclusion is reconstructed as a high inclusion in anisotropy factor. Hence, we can deduce that the two parameters $\mu_{s}$ and $g$ doesn't have the same behavior and effect on the backscattered light. These parameters present an opposite sensitivities on the reflectance [6, 43]. In other words, an increase in the reflectance could be caused by either a decrease in $g$ factor or an increase in $\mu_{s}$ coefficient, and vice-versa. In case 1 for example, the increase of the reflectance due to the inclusion B (low anisotropy factor $g^{*}=0.85$ ) has resulted in low heterogeneity in $g($ Fig. $5 b)$ and highly scattering inclusion (crosstalk) (Fig. 5a). The reconstructed results are in agreement with the previous sensitivity studies and confirm the non-uniqueness nature of the problem when several combinations of $\mu_{s}$ and $g$ can lead to identical 
boundary data.

[Table 4 about here.]

\subsubsection{Without crosstalk problem}

For computational time purpose, the simulated reconstructions for all the previous cases were performed on a relatively small phantom size. In this subsection, we consider a $2 \mathrm{~cm} \times 2 \mathrm{~cm}$ domain which contains one circular inclusion in order to test our algorithm for larger domain. The source-detectors configuration is the same as in the previous subsection in order to get closer to a realistic experimental setup based on the reflectance geometry. The synthetic data are generated on a finer triangular mesh of 21248 elements using the exact optical properties while the inversion is performed on a coarser mesh of 5312 triangular elements. The data are corrupted by $3 \%$ of noise level which is inevitable in the practical applications. The inclusion located at position $x=5 \mathrm{~mm}, y=7 \mathrm{~mm}$ is assigned the exact values $\mu_{s}^{*}=6 \mathrm{~mm}^{-1}$ and $g^{*}=$ 0.85 while $\mu_{a}=0.05 \mathrm{~mm}^{-1}$ is uniformly distributed inside the medium. The reconstructed results are depicted in Fig. 6 .

\section{[Figure 6 about here.]}

As shown in Figure 6, the inclusion is accurately identified in the exact location for both optical properties. It is also seen that the reconstructed profils of $\mu_{s}$ and $g$ along the cross-section on $y=7 \mathrm{~mm}$ are spatially well fitted with the exact solution (Figs. $6(\mathrm{c}, \mathrm{d})$ ). Furthermore, the values of optical properties are accurately retrieved even for noisy data. Also, the background is well recovered for $\mu_{s}$ and $g$ images. These results prove that the algorithm is robust for realistic domain size and can provide a good estimation quality for both $\mu_{s}$ and $g$ in this situation. However, if the exact values $\mu_{s}^{*}$ and $g^{*}$ of the inclusion are both either high or low with respect to the background values, the quality reconstruction will be relatively worse. This behavior is due to the opposite sensitivities of the two parameters on the boundary data, as deduced previously. The residual errors induced by this inclusion will be compensated yielding to small objective function level and therefore to image inaccuracy. Hence, a suitable regularization technique for the proposed algorithm is needed in order to better separate and estimate simultaneously the optical properties $\mu_{s}$ and $g$.

\section{Summary}

Reconstructions of spatial distributions of the optical properties for different cases were presented. The radiative transfer equation was used as forward model in frequency-domain and solved accurately by the MFVM. For the inversion, the gradient of the objective function with respect to $\mu_{a}, \mu_{s}$ and $g$ was computed fastly and efficiently by using the adjoint 
method. The simultaneous reconstructions of $\mu_{a}$ and $\mu_{s}$ were achieved with reasonable accuracy for lowly and even for highly absorbing media. It has also been pointed out that the estimation and crosstalk errors depend on both the inclusion location and contrast level. The main contribution of this study is the reconstruction of the spatial distribution of the anisotropy factor $g$. This estimation of $g$ was possible by using forward and adjoint models based on the RTE. Consequently, this work might open the possibility to image $g$ in tissues as an additional contrast agent for DOT. Also, we have shown the capability of the proposed algorithm to reconstruct simultaneously $\mu_{s}$ and $g$ even for large domain with noisy data. The crosstalk between the two parameters has been considered and clearly observed in both optical images. We deduced that $\mu_{s}$ and $g$ have an opposite sensitivities and effects on the reflectance. Therefore, a suitable regularization technique will be implemented as a next step, in order to reduce the strong crosstalk issue between $\mu_{s}$ and $g$. This work was a necessary preliminary study before extending the present algorithm to $3 \mathrm{D}$ reconstructions. 


\section{Bibliography}

[1] Regine Choe, Alper Corlu, Kijoon Lee, Turgut Durduran, Soren D. Konecky, Monika Grosicka-Koptyra, Simon R. Arridge, Brian J. Czerniecki, Douglas L. Fraker, Angela DeMichele, Britton Chance, Mark A. Rosen, and Arjun G. Yodh. Diffuse optical tomography of breast cancer during neoadjuvant chemotherapy: A case study with comparison to mri. Medical Physics, 32(4):1128-1139, 2005.

[2] Jeremy C Hebden, Adam Gibson, Rozarina Md Yusof, Nick Everdell, Elizabeth M C Hillman, David T Delpy, Simon R Arridge, Topun Austin, Judith H Meek, and John S Wyatt. Three-dimensional optical tomography of the premature infant brain. Physics in Medicine and Biology, 47(23):4155, 2002.

[3] PR Bargo, SA Prahl, TT Goodell, RA Sleven, G Koval, G Blair, and SL Jacques. In vivo determination of optical properties of normal and tumor tissue with white light reflectance and an empirical light transport model during endoscopy. Journal of biomedical optics, 10(3):034018, 2005.

[4] Elena Salomatina, Brian Jiang, John Novak, and Anna N. Yaroslavsky. Optical properties of normal and cancerous human skin in the visible and near-infrared spectral range. Journal of Biomedical Optics, 11(6):064026-064026-9, 2006.

[5] Joshua B. Fishkin, Olivier Coquoz, Eric R. Anderson, Matthew Brenner, and Bruce J. Tromberg. Frequency-domain photon migration measurements of normal and malignant tissue optical properties in a human subject. Appl. Opt., 36(1):10-20, Jan 1997.

[6] Michael Chu and Hamid Dehghani. Image reconstruction in diffuse optical tomography based on simplified spherical harmonics approximation. Opt. Express, 17(26):24208-24223, Dec 2009.

[7] A N Yaroslavsky, P C Schulze, I V Yaroslavsky, R Schober, F Ulrich, and H-J Schwarzmaier. Optical properties of 
selected native and coagulated human brain tissues in vitro in the visible and near infrared spectral range. Physics in Medicine and Biology, 47(12):2059, 2002.

[8] Richard van Hillegersberg, John W. Pickering, Maurice Aalders, and Johan F. Beek. Optical properties of rat liver and tumor at $633 \mathrm{~nm}$ and $1064 \mathrm{~nm}$ : Photofrin enhances scattering. Lasers in Surgery and Medicine, 13(1):31-39, 1993.

[9] Christoph-Thomas Germer, Andre Roggan, Joerg P. Ritz, Christoph Isbert, Dirk Albrecht, Gerhard Muller, and Heinz J. Buhr. Optical properties of native and coagulated human liver tissue and liver metastases in the near infrared range. Lasers in Surgery and Medicine, 23(4):194-203, 1998.

[10] Andre Charette, Joan Boulanger, and Hyun K Kim. An overview on recent radiation transport algorithm development for optical tomography imaging. Journal of Quantitative Spectroscopy and Radiative Transfer, 109(17-18):2743 2766, 2008.

[11] S. R. Arridge, M. Schweiger, M. Hiraoka, and D. T. Delpy. A finite element approach for modeling photon transport in tissue. Medical Physics, 20(2):299-309, 1993.

[12] B W Pogue and M S Patterson. Frequency-domain optical absorption spectroscopy of finite tissue volumes using diffusion theory. Physics in Medicine and Biology, 39(7):1157, 1994.

[13] A. H. Hielscher, A. D. Klose, and K. M. Hanson. Gradient-based iterative image reconstruction scheme for timeresolved optical tomography. IEEE Transactions on Medical Imaging, 18(3):262-271, March 1999.

[14] Andreas H Hielscher, Raymond E Alcouffe, and Randall L Barbour. Comparison of finite-difference transport and diffusion calculations for photon migration in homogeneous and heterogeneous tissues. Physics in Medicine and Biology, 43(5):1285, 1998.

[15] Kui Ren, Gassan S. Abdoulaev, Guillaume Bal, and Andreas H. Hielscher. Algorithm for solving the equation of radiative transfer in the frequency domain. Opt. Lett., 29(6):578-580, Mar 2004.

[16] Hyun Keol Kim and Andre Charette. A sensitivity function-based conjugate gradient method for optical tomography with the frequency-domain equation of radiative transfer. Journal of Quantitative Spectroscopy and Radiative Transfer, 104(1):24 - 39, 2007. 
[17] O. Balima, J. Boulanger, A. Charette, and D. Marceau. New developments in frequency domain optical tomography. part i: Forward model and gradient computation. Journal of Quantitative Spectroscopy and Radiative Transfer, 112(7):1229 - 1234, 2011.

[18] T. Tarvainen, M. Vauhkonen, and S.R. Arridge. Gauss-newton reconstruction method for optical tomography using the finite element solution of the radiative transfer equation. Journal of Quantitative Spectroscopy and Radiative Transfer, 109(17-18):2767 - 2778, 2008.

[19] Hao Gao and Hongkai Zhao. A fast-forward solver of radiative transfer equation. Transport Theory and Statistical Physics, 38(3):149-192, 2009.

[20] Kui Ren, Gassan S. Abdoulaev, Guillaume Bal, and Andreas H. Hielscher. Algorithm for solving the equation of radiative transfer in the frequency domain. Opt. Lett., 29(6):578-580, Mar 2004.

[21] Mathieu Francoeur, Rodolphe Vaillon, and Daniel R Rousse. Theoretical analysis of frequency and time-domain methods for optical characterization of absorbing and scattering media. Journal of quantitative spectroscopy and radiative transfer, 93(1):139-150, 2005.

[22] Joan Boulanger and Andre Charette. Reconstruction optical spectroscopy using transient radiative transfer equation and pulsed laser: a numerical study. Journal of Quantitative Spectroscopy and Radiative Transfer, 93(1-3):325 336, 2005. Fourth International Symposium on Radiative Transfer.

[23] Fatmir Asllanaj, Ahmad Addoum, and Sylvain Contassot-Vivier. Detection of tumor-like inclusions embedded within human liver tissue using a short-pulsed near-infrared laser beam: Parallel simulations with radiative transfer equation. Journal of Quantitative Spectroscopy and Radiative Transfer, 165:1 - 11, 2015.

[24] Yaobin Qiao, Hong Qi, Qin Chen, Liming Ruan, and Heping Tan. An efficient and robust reconstruction method for optical tomography with the time-domain radiative transfer equation. Optics and Lasers in Engineering, 78:155 $164,2016$.

[25] Alexander D. Klose, Uwe Netz, Jurgen Beuthan, and Andreas H. Hielscher. Optical tomography using the timeindependent equation of radiative transfer-part 1: forward model. Journal of Quantitative Spectroscopy and Radiative Transfer, 72(5):691 - 713, 2002.

[26] Avraham Y. Bluestone, Gassan Abdoulaev, Christoph H. Schmitz, Randall L. Barbour, and Andreas H. Hielscher. Three-dimensional optical tomography of hemodynamics in the human head. Opt. Express, 9(6):272-286, Sep 2001. 
[27] Andreas H. Hielscher, Alexander Klose, David Catarious Jr., and Kenneth M. Hanson. Tomographic imaging of biological tissue by time-resolved, model-based, iterative image reconstruction. In Advances in Optical Imaging and Photon Migration, page ATuB6. Optical Society of America, 1998.

[28] Alexander D. Klose and Andreas H. Hielscher. Optical tomography using the time-independent equation of radiative transfer-part 2: inverse model. Journal of Quantitative Spectroscopy and Radiative Transfer, 72(5):715 - $732,2002$.

[29] O. Balima, J. Boulanger, A. Charette, and D. Marceau. New developments in frequency domain optical tomography. part ii: Application with a l-bfgs associated to an inexact line search. Journal of Quantitative Spectroscopy and Radiative Transfer, 112(7):1235 - 1240, 2011.

[30] Jenni Heino, Simon Arridge, Jan Sikora, and Erkki Somersalo. Anisotropic effects in highly scattering media. Phys. Rev. E, 68:031908, Sep 2003.

[31] Joan Boulanger, Fengshan Liu, and Andre Charette. Investigation on the reflection at the boundaries for reconstruction laser-based imaging. Journal of Quantitative Spectroscopy and Radiative Transfer, 104(2):238 - $247,2007$.

[32] Fatmir Asllanaj, Sylvain Contassot-Vivier, Andre Liemert, and Alwin Kienle. Radiative transfer equation for predicting light propagation in biological media: comparison of a modified finite volume method, the monte carlo technique, and an exact analytical solution. Journal of Biomedical Optics, 19(1):015002, 2014.

[33] Michel Kern. Problèmes inverses : aspects numériques. Lecture, September 2002.

[34] Simon R. Arridge and Martin Schweiger. A gradient-based optimisation scheme for optical tomography. Opt. Express, 2(6):213-226, Mar 1998.

[35] Fabien Dubot, Yann Favennec, Benoit Rousseau, and Daniel R. Rousse. Regularization opportunities for the gaussnewton and bfgs algorithms: application to the diffuse optical tomography problem. International Journal of Thermal Sciences, 98, 2013.

[36] Alexander D Klose and Andreas H Hielscher. Quasi-newton methods in optical tomographic image reconstruction. Inverse Problems, 19(2):387, 2003.

[37] M. J. Holboke, Bruce J. Tromberg, X. Li, N. Shah, J. Fishkin, D. Kidney, J. Butler, Britton Chance, and Arjun G. Yodh. Three-dimensional diffuse optical mammography with ultrasound localization in a human subject. Journal of Biomedical Optics, 5(2):237-247, 2000. 
[38] Xavier Intes and Britton Chance. Multi-frequency diffuse optical tomography. Journal of Modern Optics, 52(15):2139-2159, 2005.

[39] Mehmet Burcin Unlu, Ozlem Birgul, Roshanak Shafiiha, Gultekin Gulsen, and Orhan Nalcioglu. Diffuse optical tomographic reconstruction using multifrequency data. Journal of Biomedical Optics, 11(5):054008-054008-9, 2006.

[40] Gultekin Gulsen, Bin Xiong, Ozlem Birgul, and Orhan Nalcioglu. Design and implementation of a multifrequency near-infrared diffuse optical tomography system. Journal of Biomedical Optics, 11(1):014020-014020-10, 2006.

[41] Minming Huang, Tuqiang Xie, Nan Guang Chen, and Quing Zhu. Simultaneous reconstruction of absorption and scattering maps with ultrasound localization: feasibility study using transmission geometry. Appl. Opt., 42(19):4102-4114, Jul 2003.

[42] Huabei Jiang, Brian W. Pogue, Michael S. Patterson, Keith D. Paulsen, and Ulf L. Osterberg. Simultaneous reconstruction of optical absorption and scattering mapsin turbid media from near-infrared frequency-domain data. Opt. Lett., 20(20):2128-2130, Oct 1995.

[43] M. Marin, F. Asllanaj, and D. Maillet. Sensitivity analysis to optical properties of biological tissues subjected to a short-pulsed laser using the time-dependent radiative transfer equation. Journal of Quantitative Spectroscopy and Radiative Transfer, 133:117 - 127, 2014. 


\section{List of Figures}

1 (a) Two-dimensional $\left(4 \times 4 \mathrm{~mm}^{2}\right)$ triangular mesh (b) containing three circular inclusions $\mathrm{A}, \mathrm{B}$ and C. . .

2 Reconstructions of the scattering coefficient $\mu_{s}$ for two different source numbers. (a) One source is placed at the mid-center of the north surface (b) Four sources are used to probe the mid-center of each surface of the phantom. The solide circles indicate the exact inclusion locations. . . . . . . . . . . . . .

3 Simultaneous reconstructions of the absorption $\mu_{a}$ and the scattering $\mu_{s}$ coefficients. Left column : Reconstructed $\mu_{a}$ images. Right column : Reconstructed $\mu_{s}$ images. Top raw : Test case 1. Middle raw : Test case 2. Bottom raw : Test case 3. The solide circles indicate the exact positions while the dashed circles depict the crosstalk zones. We started the minimization using the homogeneous background optical

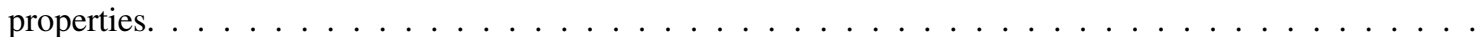

4 Reconstructions of the anisotropy factor $g$ for four different noise levels $\sigma_{m}$ on the synthetic data. The original phantom medium to reconstruct was shown in figure 1. (a) reconstructed $g$ image with noiseless data (b) reconstructed $g$ image, for $\sigma_{m}=3 \%$ (c) reconstructed $g$ image, for $\sigma_{m}=6 \%$ (d) reconstructed $g$ image, for $\sigma_{m}=10 \%$. The solide circles indicate the exact inclusion locations. . . . . . . . . . .

5 Simultaneous reconstructions of the scattering coefficient $\mu_{s}$ and the anisotropy factor $g$. Left column : Reconstructed $\mu_{s}$ images. Right column : Reconstructed $g$ images. Top raw : Test case 1, inclusion A in scattering coefficient and inclusion B in anisotropy factor. Bottom raw : Test case 2, inclusion A in anisotropy factor while inclusion B in scattering coefficient. The solide circles indicate the exact positions while the dashed circles depict the crosstalk zones. . . . . . . . . . . . . . . . . .

6 Simultaneous reconstructions of the scattering coefficient $\mu_{s}$ and the anisotropy factor $g$ for the $2 \mathrm{~cm} \times$ $2 \mathrm{~cm}$ domain. (a) : Reconstructed $\mu_{s}$ image. (b) : Reconstructed $g$ image. (c) : $\mu_{s}$ Cross-section. (d) $: g$ Cross-section. We started the minimization using the homogeneous background optical properties :

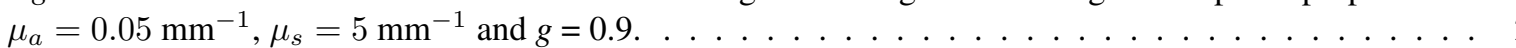


(a)

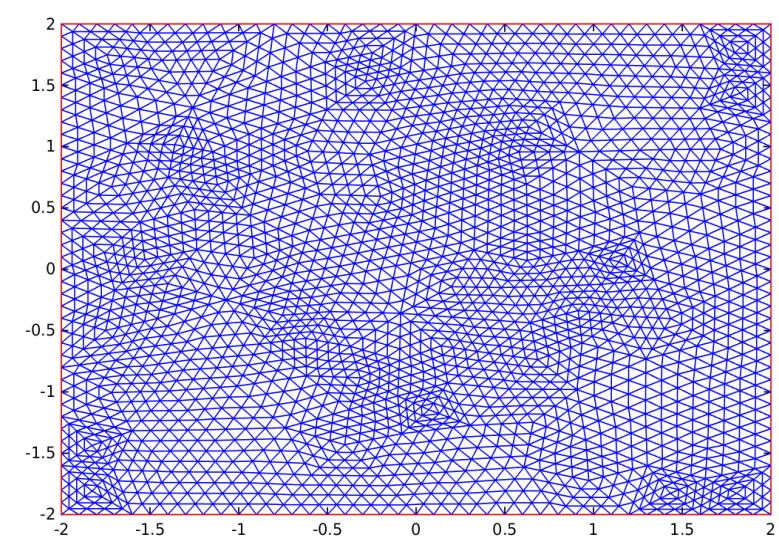

(b)

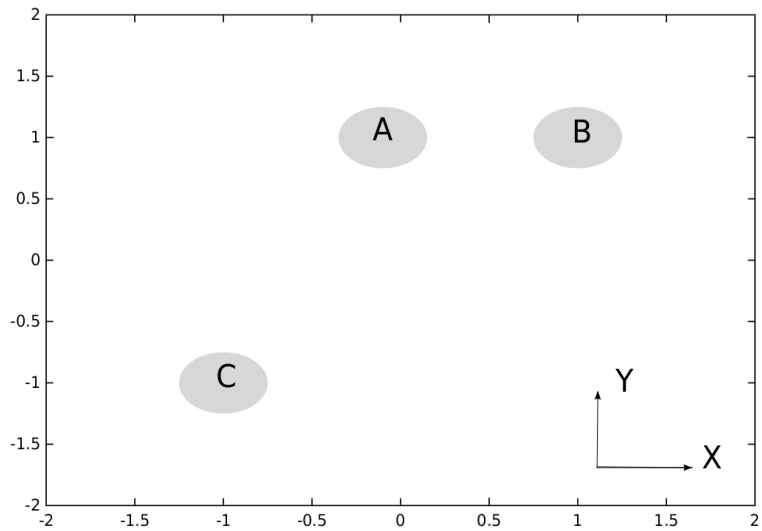

Figure 1: (a) Two-dimensional $\left(4 \times 4 \mathrm{~mm}^{2}\right)$ triangular mesh (b) containing three circular inclusions $\mathrm{A}, \mathrm{B}$ and $\mathrm{C}$. 
(a)

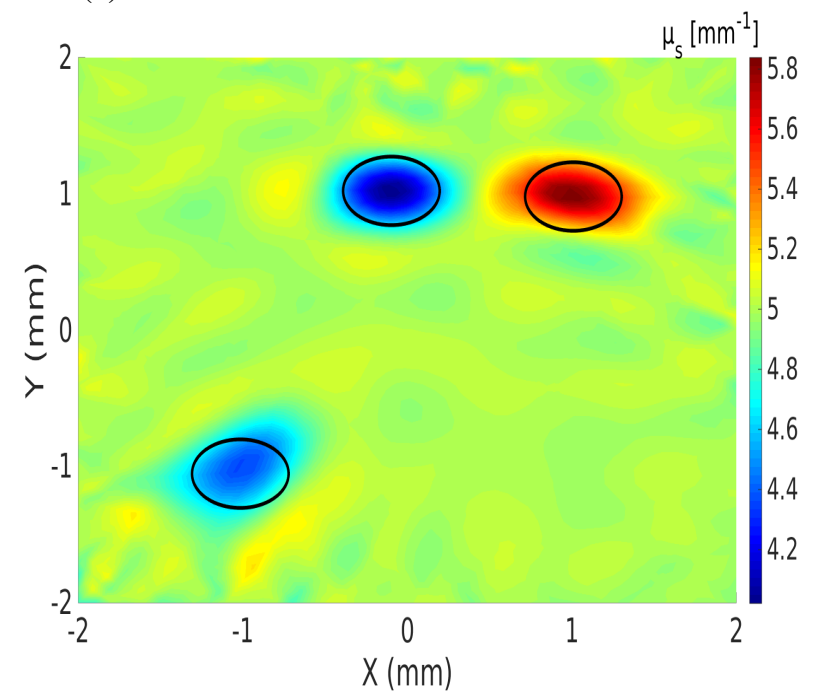

(b)

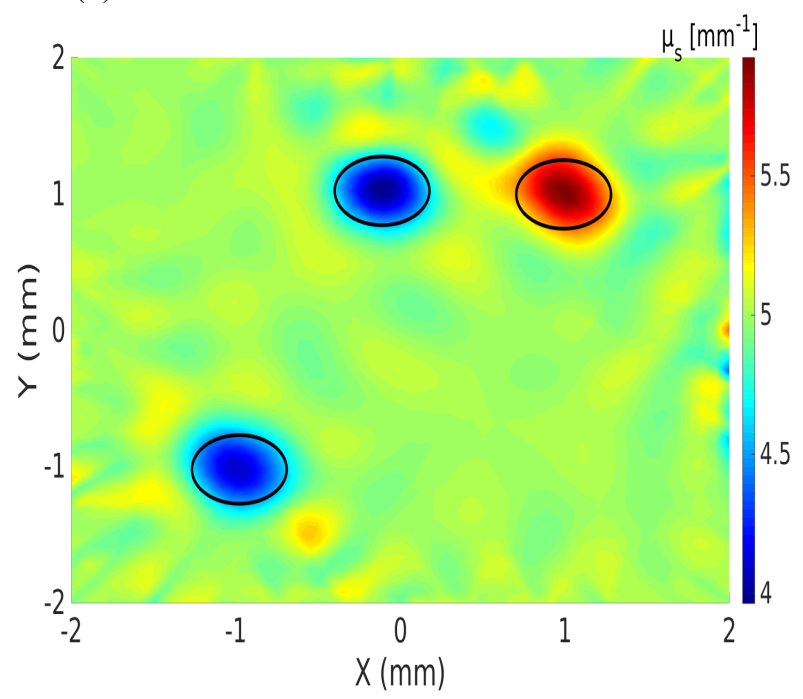

Figure 2: Reconstructions of the scattering coefficient $\mu_{s}$ for two different source numbers. (a) One source is placed at the mid-center of the north surface (b) Four sources are used to probe the mid-center of each surface of the phantom. The solide circles indicate the exact inclusion locations. 
(a)

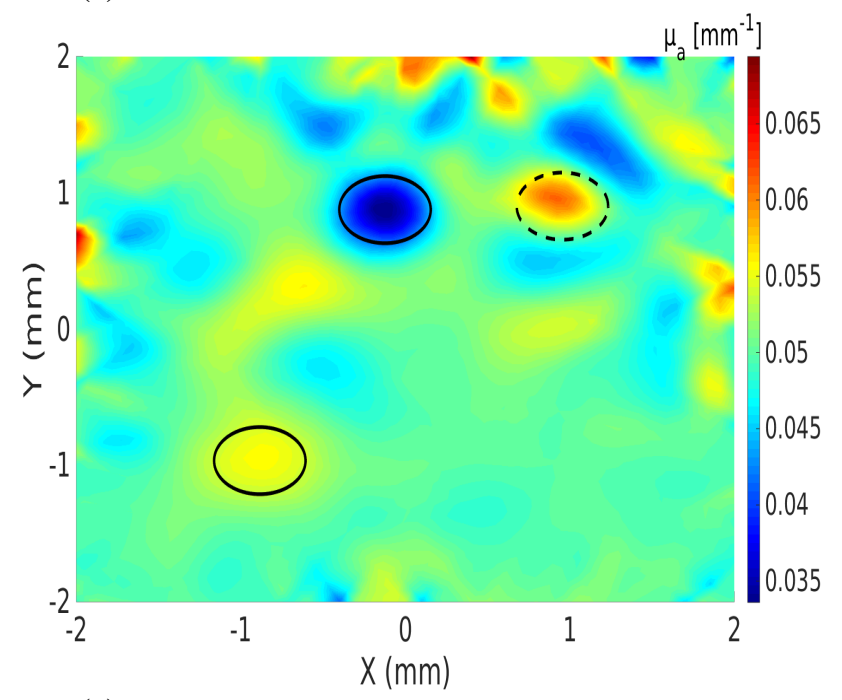

(c)

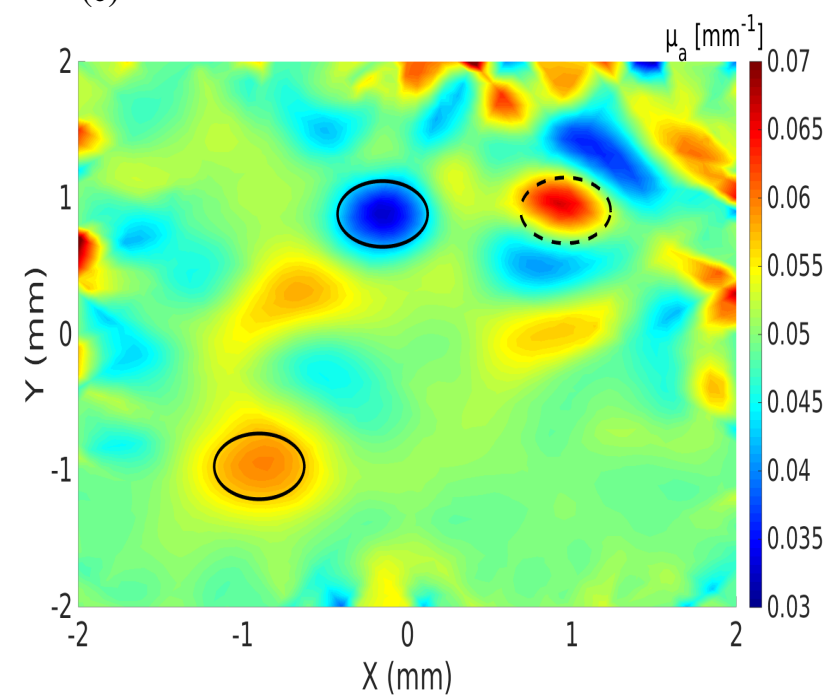

(e)

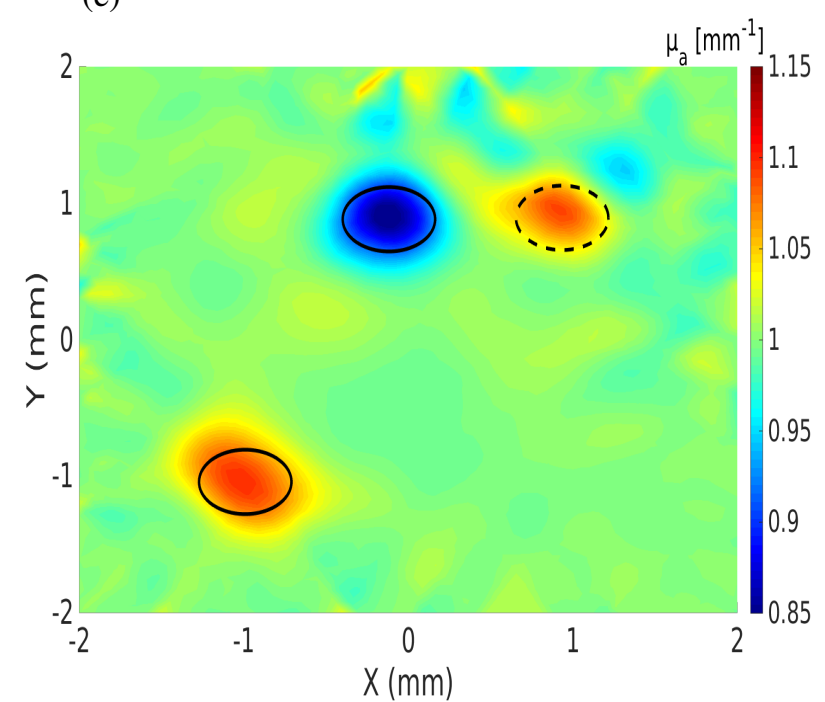

(b)

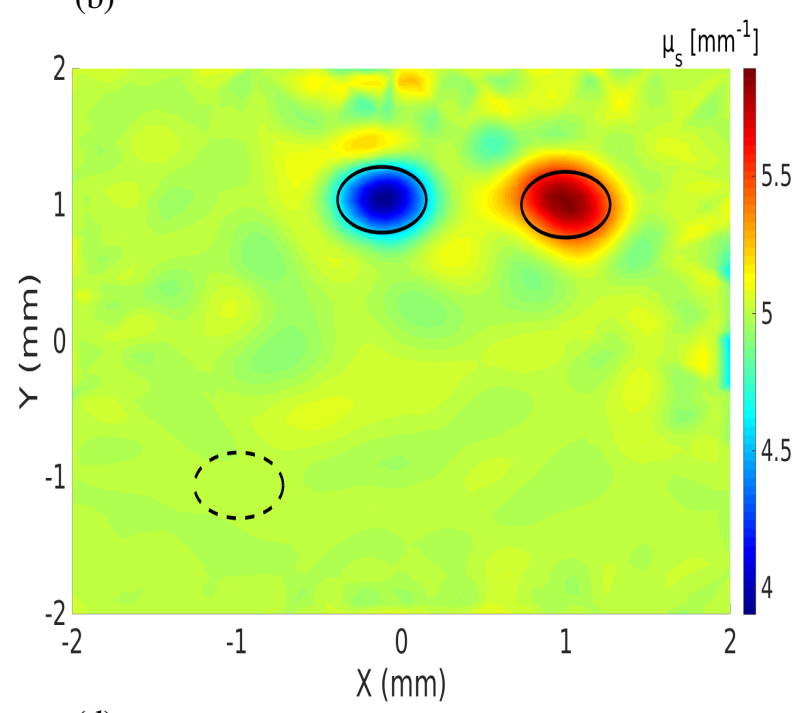

(d)

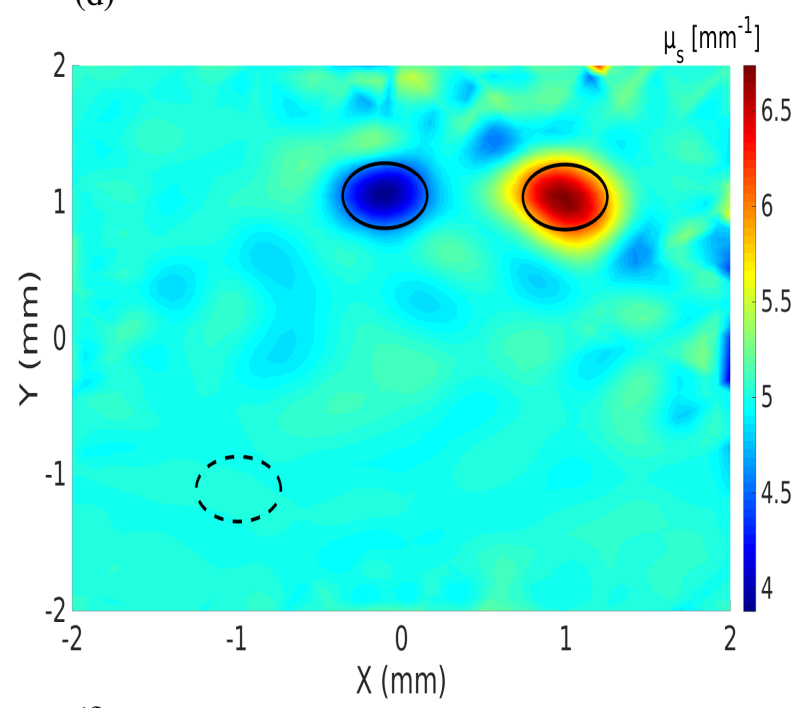

(f)

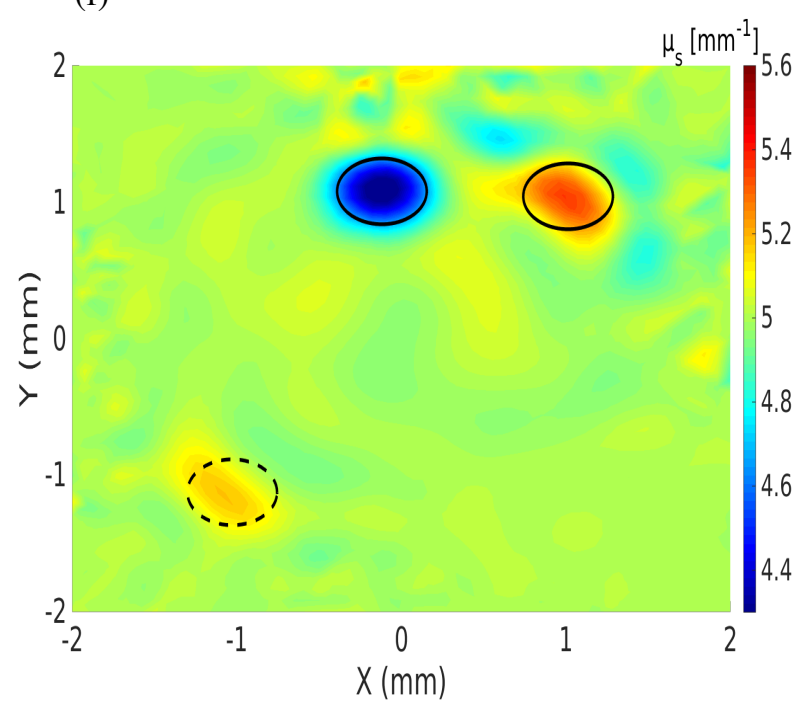

Figure 3: Simultaneous reconstructions of the absorption $\mu_{a}$ and the scattering $\mu_{s}$ coefficients. Left column : Reconstructed $\mu_{a}$ images. Right column : Reconstructed $\mu_{s}$ images. Top raw : Test case 1. Middle raw : Test case 2. Bottom raw : Test case 3. The solide circles indicate the exact positions while the dashed circles depict the crosstalk zones. We started the minimization using the homogeneous background optical properties. 
(a)

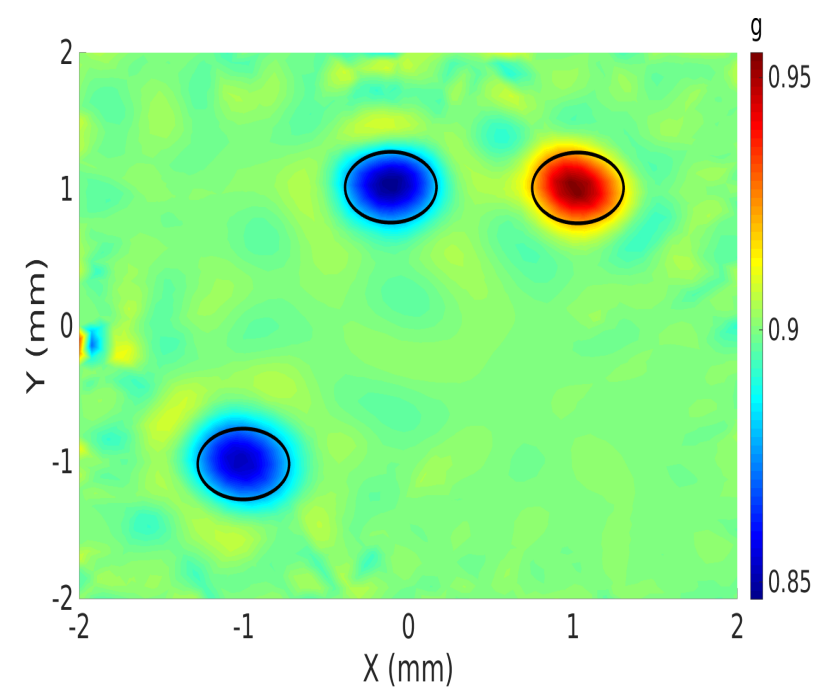

(c)

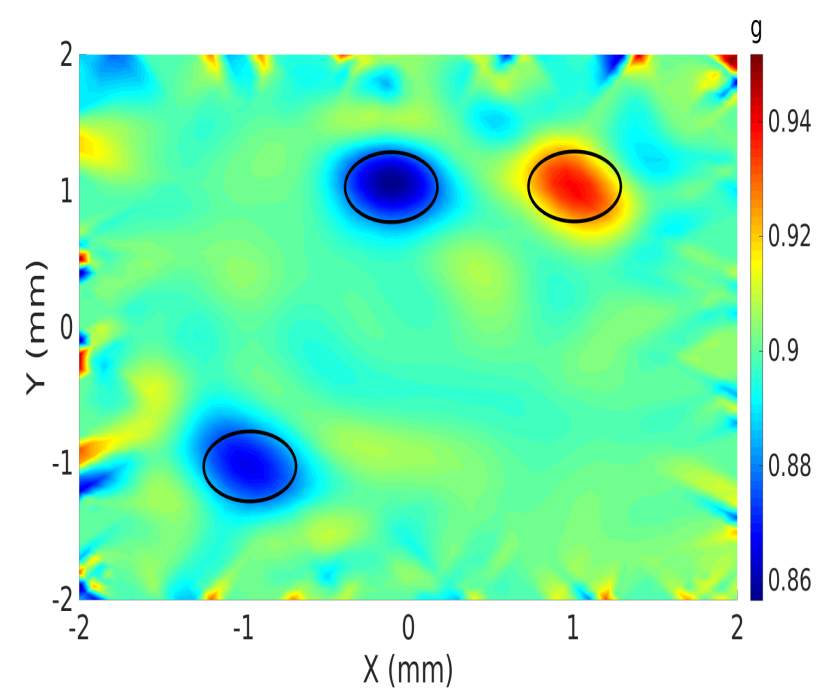

(b)

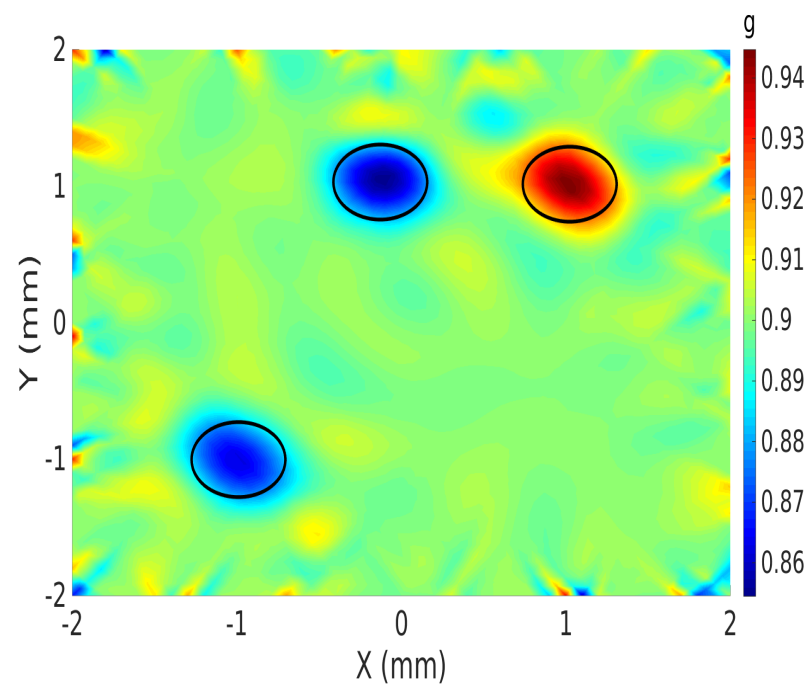

(d)

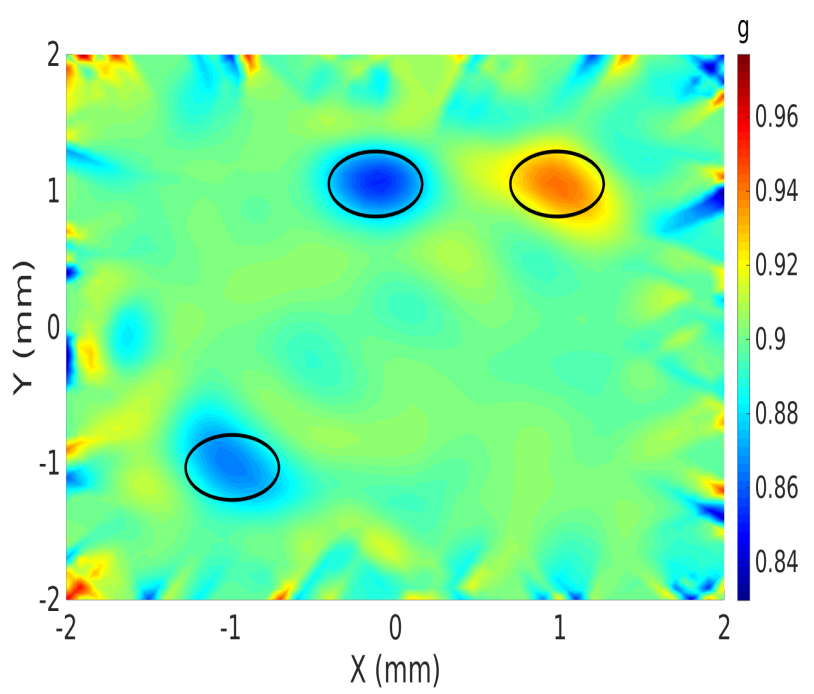

Figure 4: Reconstructions of the anisotropy factor $g$ for four different noise levels $\sigma_{m}$ on the synthetic data. The original phantom medium to reconstruct was shown in figure 1. (a) reconstructed $g$ image with noiseless data (b) reconstructed $g$ image, for $\sigma_{m}=3 \%$ (c) reconstructed $g$ image, for $\sigma_{m}=6 \%$ (d) reconstructed $g$ image, for $\sigma_{m}=10 \%$. The solide circles indicate the exact inclusion locations. 
(a)

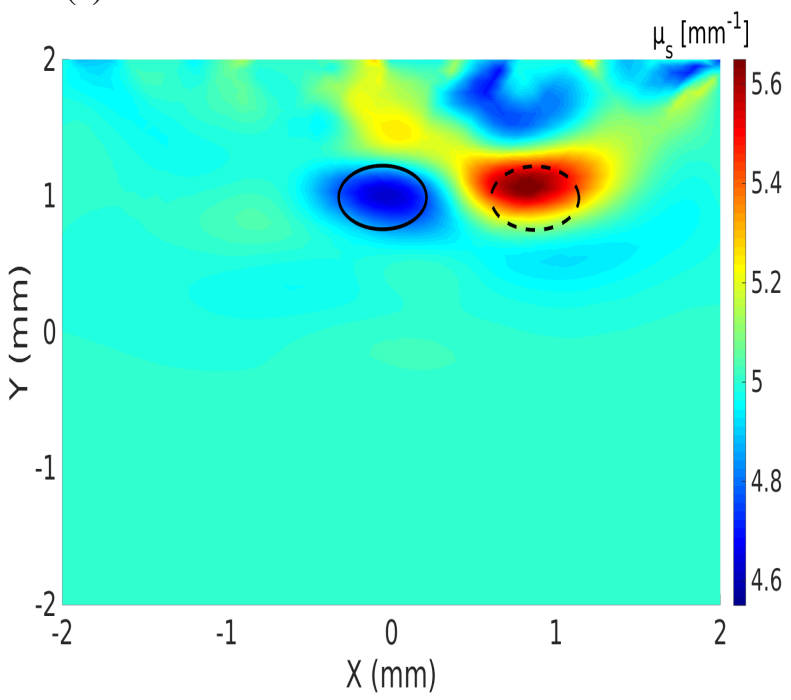

(c)

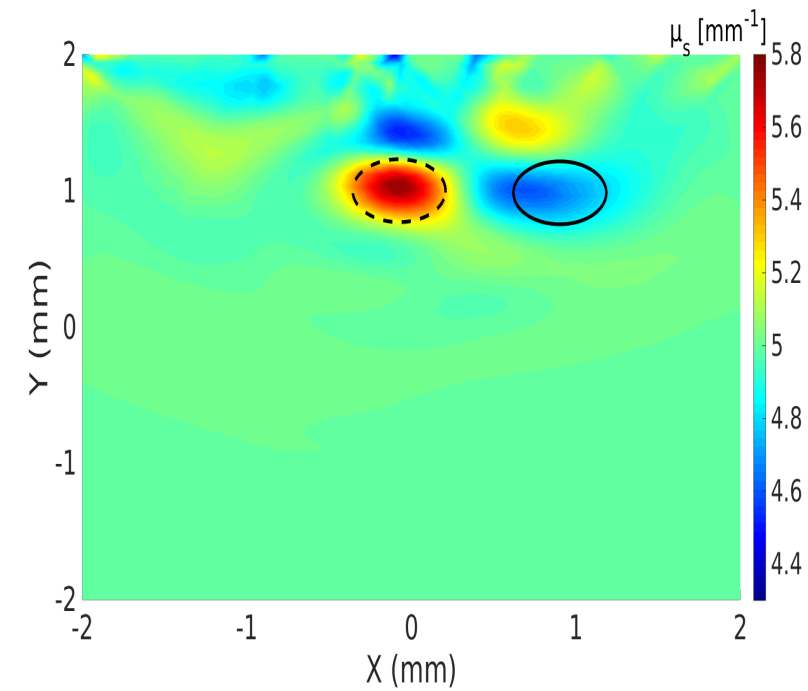

(b)

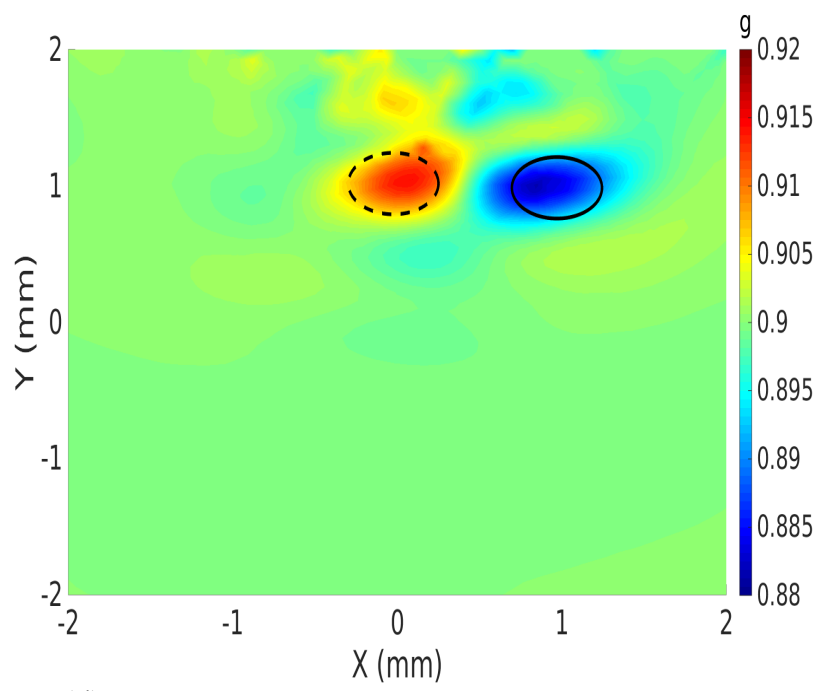

(d)

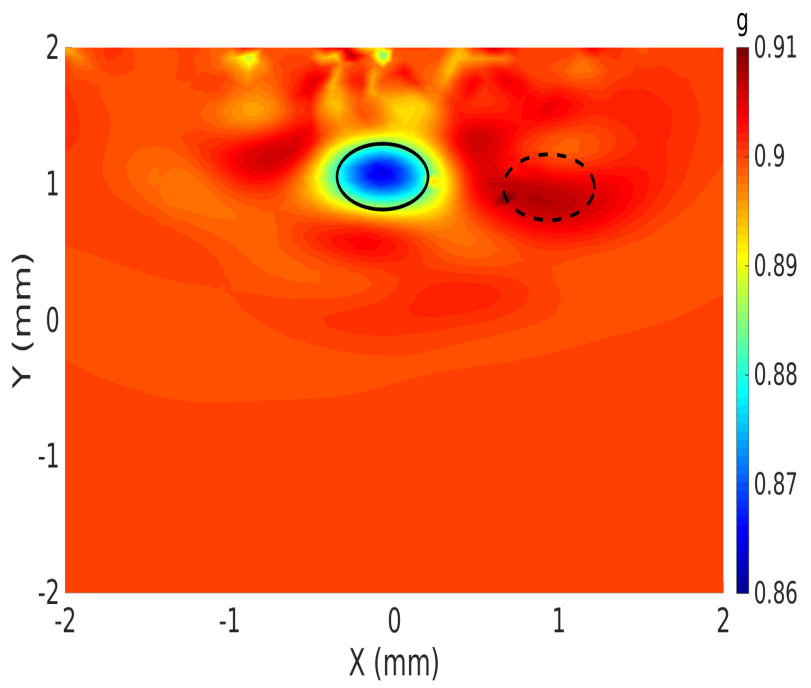

Figure 5: Simultaneous reconstructions of the scattering coefficient $\mu_{s}$ and the anisotropy factor $g$. Left column : Reconstructed $\mu_{s}$ images. Right column : Reconstructed $g$ images. Top raw : Test case 1, inclusion A in scattering coefficient and inclusion B in anisotropy factor. Bottom raw : Test case 2, inclusion A in anisotropy factor while inclusion B in scattering coefficient. The solide circles indicate the exact positions while the dashed circles depict the crosstalk zones. 
(a)

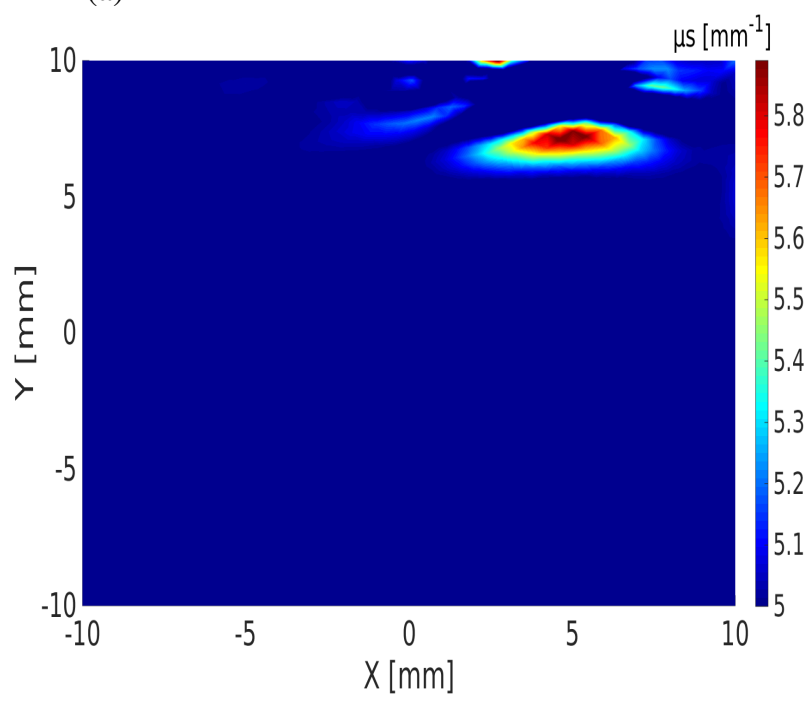

(c)

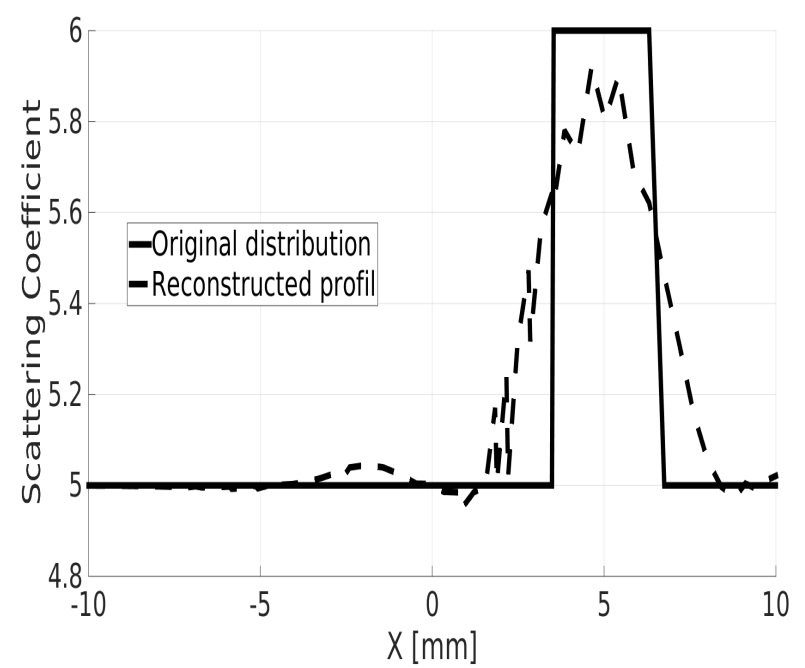

(b)

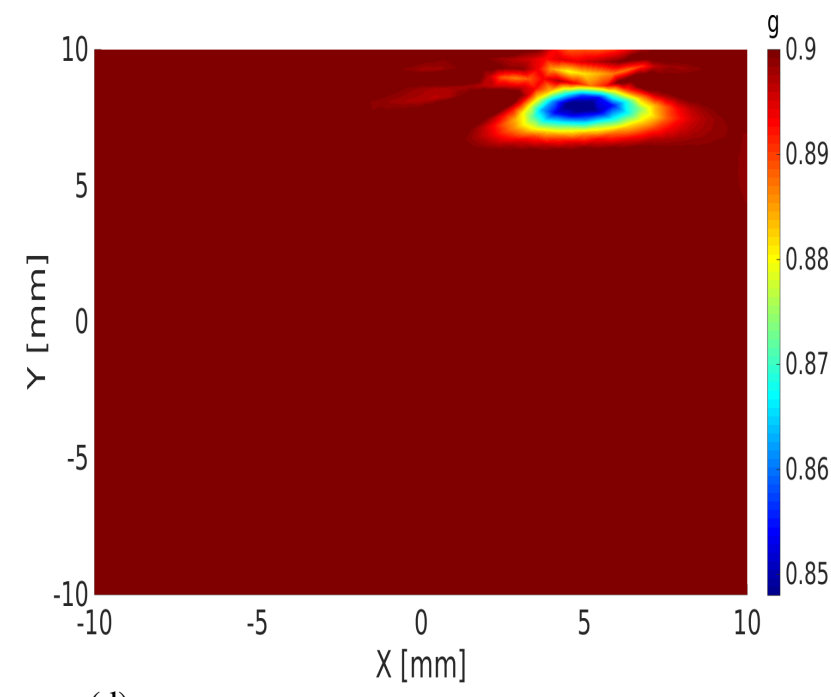

(d)

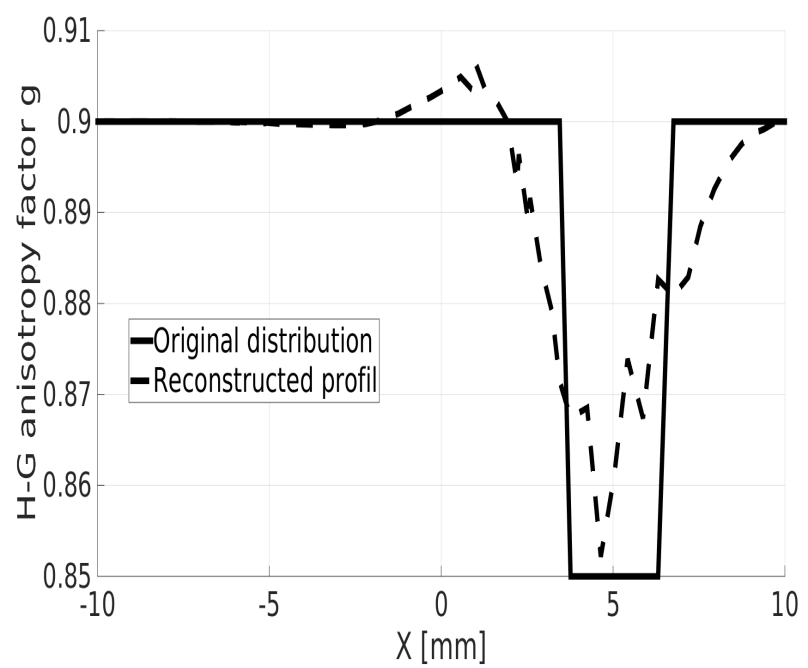

Figure 6: Simultaneous reconstructions of the scattering coefficient $\mu_{s}$ and the anisotropy factor $g$ for the $2 \mathrm{~cm} \times 2 \mathrm{~cm}$ domain. (a) : Reconstructed $\mu_{s}$ image. (b) : Reconstructed $g$ image. (c) : $\mu_{s}$ Cross-section. (d) : $g$ Cross-section. We started the minimization using the homogeneous background optical properties : $\mu_{a}=0.05 \mathrm{~mm}^{-1}, \mu_{s}=5 \mathrm{~mm}^{-1}$ and $g$ $=0.9$. 


\section{List of Tables}

1 The exact absorption and scattering coefficients of the 3 test mediums . . . . . . . . . . . 30

2 The relative estimation errors of background, inclusions and crosstalk for the absorption and scattering coefficients. . . . . . . . . . . . . . . . . . . . . . 31

3 The relative estimation errors and the iterations numbers of the reconstruction algorithm for the 4 different noise levels. . . . . . . . . . . . . . . . . . . . . . . . . . . . . 32

4 The relative estimation errors of background, inclusions and crosstalk for the scattering coefficient $\mu_{s}$ and

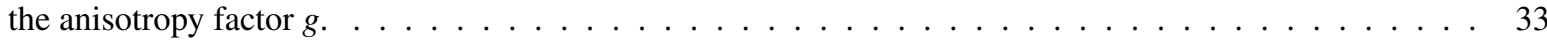




\begin{tabular}{cccccc}
\cline { 2 - 6 } & & \multicolumn{4}{c}{ Inclusions } \\
\cline { 2 - 6 } & & Background & $\mathrm{A}$ & $\mathrm{B}$ & $\mathrm{C}$ \\
\hline Test case 1 & $\mu_{a}\left(\mathrm{~mm}^{-1}\right)$ & 0.05 & 0.04 & 0.05 & 0.06 \\
& $\mu_{s}\left(\mathrm{~mm}^{-1}\right)$ & 5 & 4 & 6 & 5 \\
\hline Test case 2 & $\mu_{a}\left(\mathrm{~mm}^{-1}\right)$ & 0.05 & 0.04 & 0.05 & 0.07 \\
& $\mu_{s}\left(\mathrm{~mm}^{-1}\right)$ & 5 & 4 & 7 & 5 \\
\hline Test case 3 & $\mu_{a}\left(\mathrm{~mm}^{-1}\right)$ & 1 & 0.8 & 1 & 1.2 \\
& $\mu_{s}\left(\mathrm{~mm}^{-1}\right)$ & 5 & 4 & 6 & 5 \\
\hline
\end{tabular}

Table 1: The exact absorption and scattering coefficients of the 3 test mediums 


\begin{tabular}{c|cccc|cccc}
\hline & \multicolumn{4}{c}{$\mu_{a}$} & \multicolumn{4}{c}{$\mu_{s}$} \\
\hline & $\varepsilon_{\mu_{a}}^{\text {background }}$ & $\varepsilon_{\mu_{a}}^{\text {IncA }}$ & $\varepsilon_{\mu_{a}}^{\text {IncC }}$ & $\varepsilon_{\mu_{a}}^{\text {Crosstalk }}$ & $\varepsilon_{\mu_{s}}^{\text {background }}$ & $\varepsilon_{\mu_{s}}^{\text {IncA }}$ & $\varepsilon_{\mu_{s}}^{\text {IncB }}$ & $\varepsilon_{\mu_{s}}^{\text {Crosstalk }}$ \\
\hline Test medium 1 & $3.57 \%$ & $10.42 \%$ & $9.16 \%$ & $12.29 \%$ & $0.75 \%$ & $6.88 \%$ & $5.46 \%$ & $0.17 \%$ \\
Test medium 2 & $6.11 \%$ & $10.95 \%$ & $17.7 \%$ & $24.49 \%$ & $1.36 \%$ & $7.35 \%$ & $9.70 \%$ & $0.27 \%$ \\
Test medium 3 & $0.94 \%$ & $11.62 \%$ & $9.94 \%$ & $5.769 \%$ & $0.60 \%$ & $12.1 \%$ & $12.6 \%$ & $1.85 \%$ \\
\hline
\end{tabular}

Table 2: The relative estimation errors of background, inclusions and crosstalk for the absorption and scattering coefficients. 


\begin{tabular}{ccccc}
\hline & \multicolumn{4}{c}{ Errors } \\
\hline & $\sigma_{m}=0 \%$ & $\sigma_{m}=3 \%$ & $\sigma_{m}=6 \%$ & $\sigma_{m}=10 \%$ \\
\hline Relative estimation error $\epsilon_{g}(\%)$ & 0.29 & 0.41 & 0.53 & 0.72 \\
Number of iterations $k$ & 57 & 22 & 17 & 15 \\
\hline
\end{tabular}

Table 3: The relative estimation errors and the iterations numbers of the reconstruction algorithm for the 4 different noise levels. 


\begin{tabular}{ccccc|cccc}
\hline & \multicolumn{4}{c}{$\mu_{s}$} & \multicolumn{4}{c}{$g$} \\
\hline & $\varepsilon_{\mu_{s}}^{\text {background }}$ & $\varepsilon_{\mu_{s}}^{\text {IncA }}$ & $\varepsilon_{\mu_{s}}^{\text {IncB }}$ & $\varepsilon_{\mu_{s}}^{\text {Crosstalk }}$ & $\varepsilon_{g}^{\text {background }}$ & $\varepsilon_{g}^{\text {IncA }}$ & $\varepsilon_{g}^{\text {IncB }}$ & $\varepsilon_{g}^{\text {Crosstalk }}$ \\
\hline Test case 1 & $0.62 \%$ & $17.20 \%$ & - & $8.62 \%$ & $0.08 \%$ & - & $4.35 \%$ & $1.03 \%$ \\
Test case 2 & $0.67 \%$ & - & $19 \%$ & $9.66 \%$ & $0.1 \%$ & $3 \%$ & - & $0.49 \%$ \\
\hline
\end{tabular}

Table 4: The relative estimation errors of background, inclusions and crosstalk for the scattering coefficient $\mu_{s}$ and the anisotropy factor $g$. 